\title{
Endoplasmic reticulum stress implicated in chronic traumatic encephalopathy
}

\author{
Brandon P. Lucke-Wold, BS, ${ }^{1,2}$ Ryan C. Turner, MD, PhD, ${ }^{1,2}$ Aric F. Logsdon, BS, 2,3 \\ Linda Nguyen, BS, ${ }^{2,3}$ Julian E. Bailes, MD, ${ }^{4}$ John M. Lee, MD, PhD, ${ }^{4}$ Matthew J. Robson, PhD, ${ }^{3,5}$ \\ Bennet I. Omalu, MD, ${ }^{6}$ Jason D. Huber, PhD, ${ }^{2,3}$ and Charles L. Rosen, MD, PhD ${ }^{1,2}$ \\ 'Department of Neurosurgery, West Virginia University School of Medicine; ${ }^{2}$ The Center for Neuroscience, West Virginia

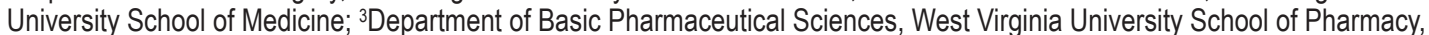 \\ Morgantown, West Virginia; ${ }^{4}$ Department of Neurosurgery, NorthShore University HealthSystem, University of Chicago Pritzker \\ School of Medicine, Evanston, Illinois; ${ }^{5}$ Department of Pharmacology, School of Medicine, Vanderbilt University, \\ Nashville, Tennessee; and ${ }^{6}$ Department of Medical Pathology and Laboratory Medicine, UC Davis Medical Center, \\ Sacramento, California
}

OBJECTIVE Chronic traumatic encephalopathy is a progressive neurodegenerative disease characterized by neurofibrillary tau tangles following repetitive neurotrauma. The underlying mechanism linking traumatic brain injury to chronic traumatic encephalopathy has not been elucidated. The authors investigate the role of endoplasmic reticulum stress as a link between acute neurotrauma and chronic neurodegeneration.

METHODS The authors used pharmacological, biochemical, and behavioral tools to assess the role of endoplasmic reticulum stress in linking acute repetitive traumatic brain injury to the development of chronic neurodegeneration. Data from the authors' clinically relevant and validated rodent blast model were compared with those obtained from postmortem human chronic traumatic encephalopathy specimens from a National Football League player and World Wrestling Entertainment wrestler.

RESULTS The results demonstrated strong correlation of endoplasmic reticulum stress activation with subsequent tau hyperphosphorylation. Various endoplasmic reticulum stress markers were increased in human chronic traumatic encephalopathy specimens, and the endoplasmic reticulum stress response was associated with an increase in the tau kinase, glycogen synthase kinase-3 $\beta$. Docosahexaenoic acid, an endoplasmic reticulum stress inhibitor, improved cognitive performance in the rat model 3 weeks after repetitive blast exposure. The data showed that docosahexaenoic acid administration substantially reduced tau hyperphosphorylation $(t=4.111, p<0.05)$, improved cognition $(t=6.532, p$ $<0.001)$, and inhibited C/EBP homology protein activation $(t=5.631, p<0.01)$. Additionally the data showed, for the first time, that endoplasmic reticulum stress is involved in the pathophysiology of chronic traumatic encephalopathy.

CONCLUSIONS Docosahexaenoic acid therefore warrants further investigation as a potential therapeutic agent for the prevention of chronic traumatic encephalopathy.

http://thejns.org/doi/abs/10.3171/2015.3.JNS141802

KEY WORDS chronic traumatic encephalopathy; blast traumatic brain injury; endoplasmic reticulum stress; hyperphosphorylated tau; docosahexaenoic acid; trauma

ABBREVIATIONS ATF6 = activating transcription factor 6; $\mathrm{BiP}=$ binding immunoglobulin protein; $\mathrm{CHOP}=\mathrm{C} / \mathrm{EBP}$ homology protein; $\mathrm{CTE}=$ chronic traumatic encephalopathy; DAB = diaminobenzidine; DHA = docosahexaenoic acid; ER = endoplasmic reticulum; GADD34 = growth arrest and DNA damage-inducible protein 34; GSK3 $\beta$ = glycogen synthase kinase-3 $\beta$; IHC = immunohistochemistry; IRE1 $\alpha=$ inositol requiring enzyme $1 \alpha ; \mathrm{JNK}=\mathrm{c}$-Jun N-terminal kinase; NFL = National Football League; PBS = phosphate-buffered saline; PERK = protein kinase RNA-like ER kinase; PHF = paired helical filament; $p$-elF2a = phosphorylated eukaryotic initiation factor $2 \alpha$; TBI = traumatic brain injury; WWE = World Wrestling Entertainment; XBP1 = X-box binding protein 1.

SUBMITTED August 6, 2014. ACCEPTED March 30, 2015.

INCLUDE WHEN CITING Published online September 18, 2015; DOI: 10.3171/2015.3.JNS141802. 
C HRONIC traumatic encephalopathy (CTE) is a progressive tau-dependent neurodegenerative disease associated with a history of concussive and/or subconcussive injury in sports or from exposure to blast waves in military personnel. ${ }^{50}$ Several progressive neuropsychiatric symptoms such as impulsive behavior, depression, loss of executive function, and cognitive decline have been reported with this progressive tauopathy. ${ }^{26}$ Alarmingly, the number of sports-related concussions has risen to 300,000 per year in the United States, and the number of military service personnel exposed to blast traumatic brain injury (TBI) during the recent wars in Iraq and Afghanistan was estimated at 270,000. ${ }^{33,49}$ Although several case reports and case series have examined the underlying pathology of CTE, few studies have looked at pathways that link TBI to chronic neurodegeneration. ${ }^{27,35,36,55}$ As such, the pathophysiology of CTE development remains poorly understood and no viable therapeutics are under development due to the lack of identified therapeutic targets. ${ }^{58}$ To address this lack of understanding, we investigated one component of the pathophysiology linking acute neurotrauma to chronic neurodegeneration.

We propose that the endoplasmic reticulum (ER) stress response may play an important role in linking acute neurotrauma to chronic tau-dependent neurodegeneration. ${ }^{24}$ The ER stress response consists of three arms: protein kinase RNA-like ER kinase (PERK), inositol requiring enzyme $1 \alpha(\mathrm{IRE} 1 \alpha)$, and activating transcription factor 6 (ATF6). The arms are activated when binding immunoglobulin protein (BiP) dissociates from the ER membrane, signaling an increase in unfolded proteins. ${ }^{18}$ While Arm one (PERK) is generally neuroprotective, it can elevate $\mathrm{C} / \mathrm{EBP}$ homology protein (CHOP) above the threshold at extended time points, which lead to apoptosis. ${ }^{16}$ Arms two (IRE1 $\alpha$ ) and three (ATF6) likewise increase CHOP and trigger proapoptotic pathways. ${ }^{45}$ Arm two can also lead to the phosphorylation of c-Jun N-terminal kinase (JNK) ${ }^{60}$ ER stress markers are acutely increased following TBI in rodent models. ${ }^{21}$ Recent findings have shown that ER stress increases following cortical contusion in a rat model, with changes in amyloid precursor protein and tau. ${ }^{4}$ Weight-drop-induced TBI has been shown to increase intracellular calcium and result in the activation of the ER stress response in mice. ${ }^{43}$ Controlled cortical impact also increases ER stress and triggers apoptosis in rats acutely after injury. ${ }^{11}$ While these studies demonstrate the role of ER stress in acute pathophysiological events after TBI, how ER stress contributes to persistent neurodegeneration remains unclear. Abisambra and colleagues have ascertained that tau from neurofibrillary tangles perpetually activates the ER stress response. ${ }^{1}$ Conversely the ER stress pathway can activate tau kinases such as apoptosis signal-regulating kinase $1, \mathrm{JNK}$, and glycogen synthase kinase- $3 \beta$ (GSK3 $\beta)^{8,38,48}$ These important costimulatory interactions connect ER stress in interplay with chronic neurodegenerative diseases. In addition, phosphorylated PERK has been colocalized with tau in diseases such as Alzheimer's disease and supranuclear palsy. ${ }^{52}$

Herein, we provide compelling evidence that the ER stress response is not only activated acutely following neurotrauma but is also involved in the development and pro- gression of CTE-like findings in an animal model and in postmortem specimens that were previously described as having CTE. ${ }^{36}$ Furthermore, we show that the development of CTE-like findings following repetitive concussive blast in rats is prevented by administration of docosahexaenoic acid (DHA). DHA, an important component of omega-3 fatty acids, has recently been shown to decrease ER stress by lowering intracellular calcium. ${ }^{4}$ Our data show that DHA administration decreases tau kinases, tau hyperphosphorylation, and improves cognitive performance 3 weeks after repetitive blast injury. Modulation of ER stress responses may therefore represent a promising preventative and therapeutic strategy in the field of neurotrauma.

\section{Methods}

\section{Animals and Human Samples}

All experiments involving animals were approved by the Institutional Animal Care and Use Committee of West Virginia University and were performed according to the principles of the Guide for the Care and Use of Laboratory Animals. Eighty-eight young-adult male rats (Hilltop Lab Animals, Inc.) were used for this study based on power analysis calculations (DSS Research). We used 24 rats for Morris water maze behavioral testing, 32 rats for immunohistochemistry (IHC) staining, and 32 rats for Western blot analysis. For human IHC studies, we used sections from the entorhinal cortex of a National Football League (NFL) player with diagnosed CTE, a World Wrestling Entertainment (WWE) wrestler with diagnosed CTE, and an age-matched (44-year-old male) control.

The case of the WWE wrestler was previously documented and published in 2010. ${ }^{36}$ Briefly, he was 40 years of age at the time of death. Death was ruled a suicidedeath by asphyxiation resulting from hanging. The patient had a history of repetitive brain trauma that included a car collision and mild TBI at 6 years of age and at least 15 self-reported concussions throughout his career in wrestling. Prior to his death, he suffered from depression and memory loss. The patient also had a history of presumed anabolic steroid use and suspected prescription drug dependency (narcotics).

The case of the NFL player was previously documented and published in $2010 .{ }^{35}$ Briefly, he was 36 years of age at the time of death, which was the result of a head-on motor vehicle collision with a tanker truck. The patient had a history of repetitive head trauma from 4 years of collegiate football and 8 seasons in the NFL. Prior to his death, he suffered from cognitive and neuropsychiatric symptoms. He also had a history of parasuicidal behavior.

\section{Experimental Groups, Blast Exposure, and Drug Treatments}

The control groups received no blast injury but were anesthetized with 4\% isoflurane. A single blast (50 PSI reflected) was administered perpendicular to the right side of the rat's skull while the animal was anesthetized (4\% isoflurane). Repeat blasts involved 6 total blasts given every other day for a total of 2 weeks, with the rats having been anesthetized prior to each blast. Groups for 24-hour postinjury IHC study were as follows: control, single blast, 
and repeat blast. Groups for 24-hour postinjury Western blot study were as follows: control, single blast, single blast+DHA, and DHA alone. Groups for 3-week postinjury Western blot and IHC study were as follows: control, repeat blast, and repeat blast+DHA. Groups for 3-week postinjury behavioral study were as follows: control, repeat blast, and repeat blast+DHA. All groups receiving repeat-blast exposure had 1 blast administered every other day for 2 weeks (6 total blasts). DHA (Zone Labs, Inc.) was delivered intraperitoneally $16 \mathrm{mg} / \mathrm{kg} 5$ minutes following each blast exposure for the DHA+repeat-blast groups, and subsequently every other day until Morris water maze testing. ${ }^{4}$

\section{Blast Exposure}

A scaled moderate-intensity blast wave(s) (approximately 50.72-PSI peak reflected over pressure) was released perpendicular to the right side of the animal's head with a shield of polyvinyl chloride protecting its body. Blast exposures were generated using a novel shock tube described in our prior work. ${ }^{54}$ The shock tube was designed with a short driver section to generate a shortduration wave, a characteristic deemed clinically relevant based on extensive work demonstrating scaling principles associated with blast across species. The overpressure was recorded using piezoelectric sensors (Model 102AO5; PCB Piezotronics) placed in the reflected and incident positions at the exit of the shock tube. Data were then recorded using a sensor signal conditioner (482C Series; PCB Piezotronics) and a data acquisition board (DAQ 23GF; National Instruments). Data were then recorded on the computer using a customized program written with LabView version 12.0 (National Instruments). A representation of the experimental setup can be seen in Fig. 1.

\section{Biochemistry Preparation}

Animals used for Western blot analysis were sacrificed by decapitation with a sharpened guillotine after being anesthetized by inhalation of $4 \%$ isoflurane (Halocarbon). For Western blot, the brains were immediately placed in a lysate buffer containing the following: $10 \mathrm{mM}$ Tris, $\mathrm{pH}$ 7.4, $320 \mathrm{mM}$ sucrose, $1 \%$ Triton X-100, 1\% CHAPS, and $0.025 \% \mathrm{NaN}_{3}$, with phosphatase and protease inhibitors (1 mM EDTA, $1 \mathrm{mM}$ EGTA, $50 \mathrm{mM}$ sodium fluoride, 2 $\mathrm{mM}$ sodium orthovanadate, $0.1 \mathrm{mM}$ ammonium molybdate, $0.2 \mathrm{mM}$ phenylarsine oxide, and Roche Complete tablets).$^{39}$ Bilateral dissections of the cortex, hippocampus, striatum, and cerebellum were performed, and tissue was subsequently flash frozen in liquid nitrogen and stored at $-80^{\circ} \mathrm{C}$ for current and future studies. Animals used for immunohistochemistry were anesthetized with $4 \%$ isoflurane (Halocarbon) and transcardially perfused with icecold $0.9 \%$ saline and then with $10 \%$ formalin for a total of 10 minutes. The brains were dissected and placed in $10 \%$ formalin. Brains were block-sectioned into 4 coronal sections, embedded in paraffin, and serially sliced with a Leica RM2235 microtome (Leica Microsystems) for immunofluorescent, diaminobenzidine, and $\mathrm{H} \& \mathrm{E}$ staining.

\section{Western Blot}

Western blot procedures were performed similarly to previous work published by our lab. ${ }^{10}$ Hippocampal protein samples were dissolved in $0.5 \mathrm{ml}$ hot $\left(85^{\circ}-95^{\circ} \mathrm{C}\right) 1 \%$ sodium dodecyl sulfate, sonicated, and assayed with a bicinchoninic acid protein assay kit (Thermo Fisher Scientific). Thirty micrograms of protein per well was run with $2 \times$ Laemmli buffer on precast Bolt Bis-Tris Plus 10\% 12-well gels (Life Technologies) using a Bolt Mini tank system (Life Technologies). A SeeBlue Plus2 Prestained Standard (Life Technologies) was used. Nitrocellulose membranes (Bio-Rad) were used for wet transfer (Bio$\mathrm{Rad})$ at $60 \mathrm{~V}$ for 2.5 hours. Imaging was conducted with a LI-COR fluorescent scanner at wavelength 700 or 800 , intensity of 6.0, and resolution of 84. Images were converted to gray scale and analyzed after background subtraction (Odyssey Processing Software). Values were normalized to $\beta$-actin to give relative overall intensity.

The following primary antibodies were used: AT270, growth arrest and DNA damage-inducible protein 34 (GADD34; Thermo), BiP, CHOP, p-JNK (Cell Signaling), p-GSK3 $\beta$ (Santa Cruz), and CP-13 (kindly gifted by Dr. Peter Davies, Albert Einstein College of Medicine), all at a concentration of 1:1000. Anti-rabbit or anti-mouse IRDye secondary antibodies (LI-COR) were used at a concentration of 1:2000. A $\beta$-actin rabbit monoclonal antibody (Cell Signaling) was used as an endogenous control at a concentration of 1:10,000. Imaging was conducted with a LI-COR fluorescent scanner, and images were analyzed after background subtraction (Odyssey Processing Software). Values were normalized to $\beta$-actin to give relative overall intensity.

\section{Immunohistochemistry}

Qualitative and quantitative staining was completed using previously described techniques for $\mathrm{H} \& \mathrm{E}$, brightfield imaging with diaminobenzidine (DAB), and fluorescent imaging. ${ }^{41,54}$ Briefly, to remove paraffin, slides were washed for 5 minutes in xylene, $100 \% \mathrm{EtOH}$, and $95 \%$ EtOH followed by 5 -minute rehydration in $\mathrm{dH}_{2} \mathrm{O}$. The slides were then quenched with $10 \%$ methanol and $10 \%$ $\mathrm{H}_{2} \mathrm{O}_{2}$ in Dulbecco's phosphate-buffered saline (PBS) for 15 minutes. After quenching, slides were rinsed 2 times in PBS for 10 minutes each. The slides were then placed in permeabilizing solution (1.8\% L-Lysine, $4 \%$ horse serum, and $0.2 \%$ Triton X-100 in PBS) for 30 minutes. For fluorescent staining, brain slices were circumscribed, incubated with primary antibody overnight followed by a fluorescent secondary for 3 hours, and fixed with a coverslip. If staining for colocalization, a second set of primary and secondary antibody was applied prior to fixing the coverslip. For DAB staining, primary antibody, secondary antibody, streptavidin-horseradish peroxidase, and then DAB were applied before fixing with the coverslip. The $\mathrm{H} \& \mathrm{E}$ protocol consisted of dissolving off paraffin with 5-minute washes in xylene, $100 \% \mathrm{EtOH}$, and $95 \% \mathrm{EtOH}$. The slides were then stained in Harris hematoxylin solution for 2 minutes and 15 seconds. The slides were rinsed in running tap water and then dipped in differentiating solution $(0.25 \mathrm{ml}$ concentrated $\mathrm{HCl}$ in $100 \mathrm{ml}$ of $70 \% \mathrm{EtOH})$. The slides were rinsed again in running tap water and placed in Scott's Tap Water substitute $\left(2 \mathrm{~g}\right.$ of $\mathrm{NaHCO}_{3}$ and $20 \mathrm{~g}$ of $\mathrm{MgSO}_{4}$ in $1 \mathrm{~L}$ of tap water) for 1 minute. Slides were 

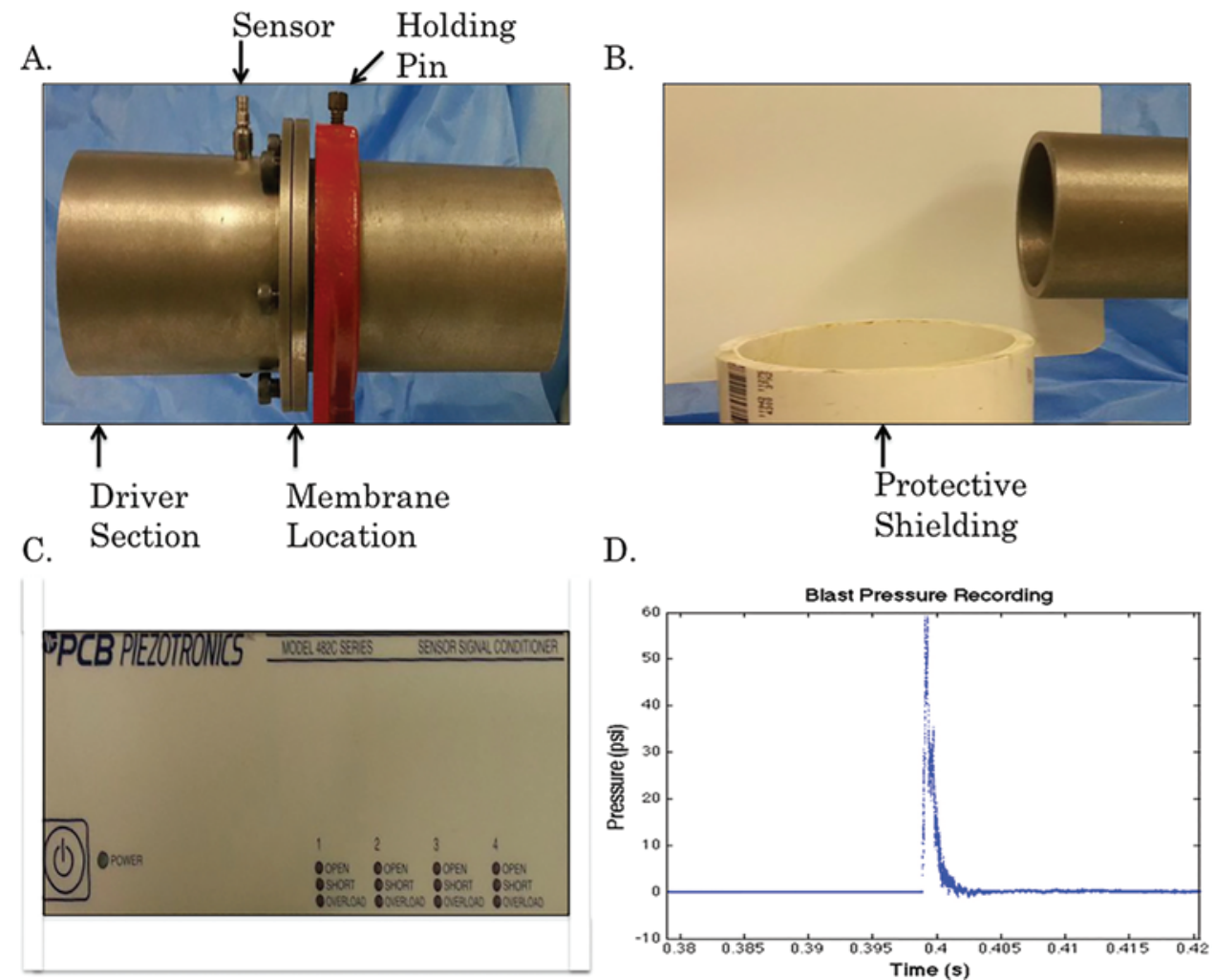

FIG. 1. Representative setup of the blast model. A: The blast model with piezoelectric sensor and driver section. B: A polyvinyl tube protects the animal's body from blast wave. C: The sensor signal conditioner. D: Graph showing peak overpressure recorded from an approximately 50-PSI blast wave. Figure is available in color online only.

placed in $95 \% \mathrm{EtOH}$ for 0.5 minutes and stained with Eosin Y solution for 0.5 minutes. The slides were dehydrated with 5-minute washes in $95 \% \mathrm{EtOH}, 100 \% \mathrm{EtOH}$, and xylene and then a coverslip was fixed with Permount.

Primary antibodies were CHOP, IRE1 $\alpha, \mathrm{X}$-box binding protein 1 (XBP1), phosphorylated eukaryotic initiation factor $2 \alpha$ (p-eIF2 $\alpha$ ), p-PERK, ATF6 (Cell Signaling), AT100, AT270, GSK3 $\beta$ (Thermo), MCI, paired helical filament (PHF), and CP-13 (kindly gifted by Dr. Peter Davies, Albert Einstein College of Medicine). Secondary antibodies for fluorescent imaging were Alexa Fluor 488 and 594 (Life Technologies) tagged with the corresponding species to the primary antibody. Imaging was done with a Zeiss Axio Imager 2 (Carl Zeiss Microscopy). For quantification of DAB staining, perivascular regions were randomly selected from the hippocampus region in rats and the entorhinal cortex in humans. An observer blinded to the experimental group randomly selected 100 total cells. The number of positive cells was reported as a fraction of total cells counted. For fluorescent staining, 20 cells per slide were randomly selected by blinded observer, outlined, and measured. Fluorescent density was compared with background readings, corrected with ImageJ software, and quantified. Colocalization quantification with the Just Another Colocalization Plugin for ImageJ was used to determine overlap coefficient or Pearson's coefficient. ${ }^{6}$ Overlap coefficient was calculated using the formula $\mathrm{k}^{\wedge} 2=\mathrm{k} 1 * \mathrm{k} 2$, with values adjusted to threshold.

\section{Morris Water Maze}

Animals were assessed beginning 2 weeks after final blast exposure. The pool was approximately $180 \mathrm{~cm}$ in diameter and had a $10 \times 10-\mathrm{cm}$ platform submerged 2.5 $\mathrm{cm}$ below the surface of the water $\left(20^{\circ} \mathrm{C}\right)$. The training paradigm consisted of 6 days of spatial acquisition with a hidden platform followed by a probe day when the platform was removed. On acquisition days, rats were placed into the maze apparatus 4 times from different predetermined locations. The animals were allowed 2 minutes per trial to locate the platform. Timing stopped once the platform was found. If the animal did not find the platform, it was placed on the platform for 15 seconds to facilitate acquisition before starting the next trial. On the probe day, animals were placed in the apparatus for 1 minute at a novel entrance point. The time the animal spent exploring an area encompassing 300\% of where the platform had been was recorded. Any-Maze Video Tracking software (Stoelting Co.) was used to record distance traveled as well as latency to reach platform for all studies. Tracking plots for each animal were recorded (Fig. 2).

\section{Statistical Analysis}

A blinded observer performed all quantification for IHC and Western blot. One-way ANOVA was used for IHC, Western blot, and Morris water maze. Bonferroni post hoc comparison was used to determine differences between groups. For DAB staining, a chi-square analysis 
A.

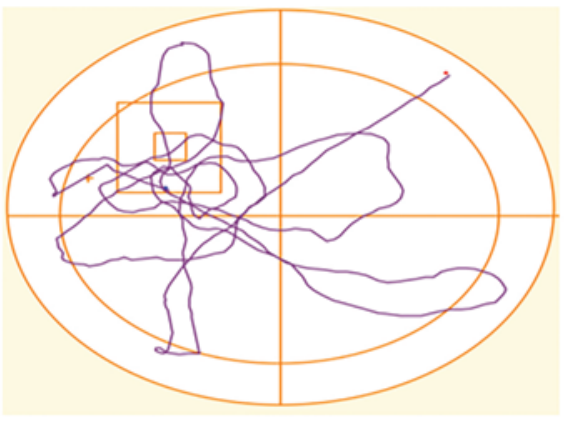

C.

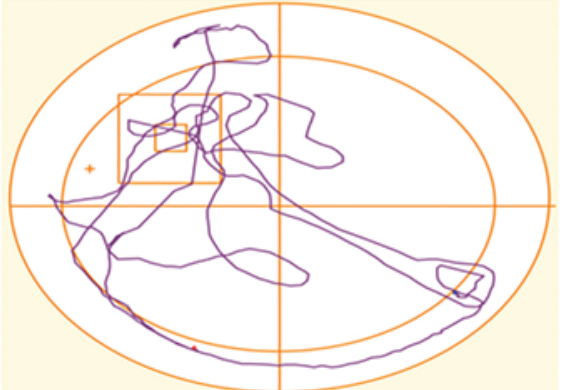

B.

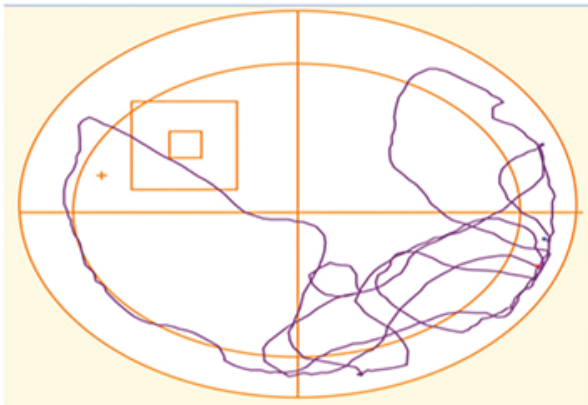

FIG. 2. Representative track plots for Morris water maze on probe trial day: track plot for control animal (A), track plot for repeatblast animal (B), and track plot for repeat-blast+DHA animal (C). Figure is available in color online only.
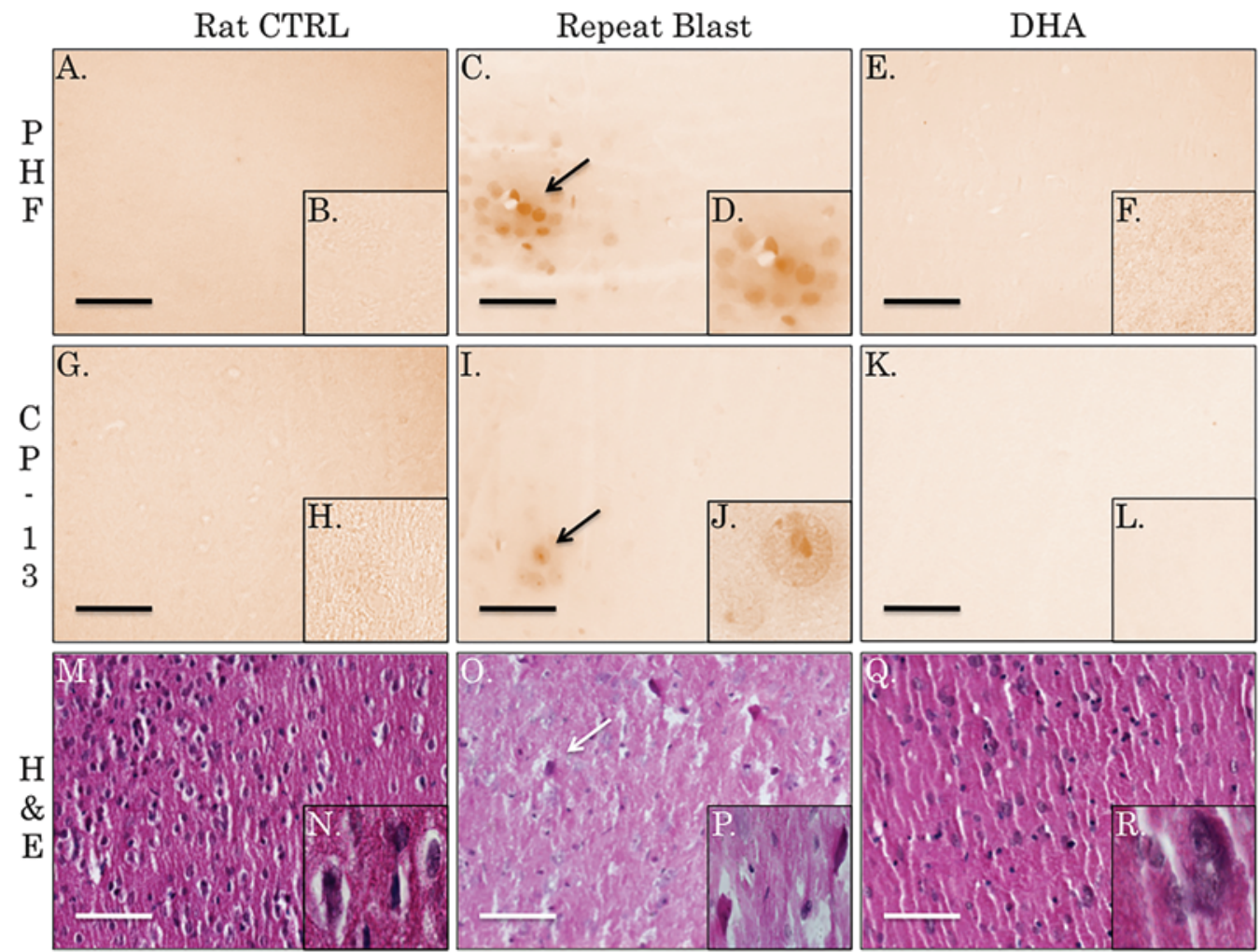

FIG. 3. Robust tau changes 3 weeks after repetitive blast exposure. Nontransgenic rodents do not develop neurofibrillary tau tangles. Tau hyperphosphorylation can occur after traumatic neurotrauma, however, indicating acute changes similar to human pathology. In the Sprague-Dawley rodent model, DAB staining revealed that PHFs were present in perivascular regions of the hippocampus for repeat-blast animals (ratio 18/100) (C and D), but not in control (ratio 0/100) (A and B), and were reduced in DHA+repeat-blast animals (ratio 3/100) (E and F). Similarly, CP-13 was increased in repeat-blast animals (ratio 17/100) (I and J), but not in control (ratio 0/100) (G and H), and was reduced in DHA+repeat-blast animals (ratio 4/100) (K and L). In addition, H \& E staining revealed nuclear chromatolysis, vacuolization, and degeneration after repeat blast ( $\mathbf{O}$ and $\mathrm{P})$, but not in control ( $\mathrm{M}$ and $\mathbf{N})$ or repeat-blast+DHA groups $(Q$ and $R)$. Original magnification $\times 20$ and bar $=100 \mu \mathrm{m}(A, C, E, G, I, K, M, O$, and $Q)$; and original magnification $\times 63(B, D, F, H, J, L, N, P$, and $R)$. CTRL = control. Figure is available in color online only. 
was used for comparison of groups. For colocalization studies, Pearson's coefficient was obtained for control sections. Overlap coefficient was obtained for experimental groups to determine the extent of same-cell protein expression. Two-way ANOVA was used to determine timedependent acquisition for Morris water maze learning trials. GraphPad Prism 5.0 (GraphPad Software, Inc.) was used for statistical analysis; $p<0.05$ was considered statistically significant for all data analyzed.

\section{Results}

\section{Tau Pathology Is Evident in Blast-Injured Animals and Human CTE}

Key pathological findings for CTE include tau hyperphosphorylation and neuritic threads. The pathology is commonly located in subcortical and perivascular foci. ${ }^{28}$ DAB staining revealed increased tau markers, PHF, and CP-13, after repeat blasts in perivascular areas adjacent to the hippocampus in Sprague-Dawley rats 3 weeks postinjury, but not in control or repeat-blast+DHA animals (Fig. $3 \mathrm{~A}-\mathrm{L}$ ). The ratio of positive PHF-stained cells was $0 / 100$ for control, 18/100 for repeat-blast, and 3/100 for repeatblast+DHA animals (chi-square test 26.571 with 2 degrees of freedom, $p<0.0001$ ). The ratio of positive CP-13stained cells for controls was $0 / 100$, for repeat blast it was $17 / 100$, and for repeat-blast+DHA it was $4 / 100$ (chi-square test 22.571 with 2 degrees of freedom, $p<0.0001$ ). In addition, $\mathrm{H} \& \mathrm{E}$ staining revealed a heterogeneous appearance, chromatolysis, vacuolization, and degenerating neurons 3 weeks after repetitive blasts but not in control or repeatblast+DHA animals (Fig. 3M-R). Likewise, DAB staining revealed increased MCI, PHF, and CP-13 in the entorhinal cortex of human subjects exposed to repeated head trauma (CTE diagnosis) as compared with specimens obtained in age-matched control without a history of head trauma (Fig. 4A-R). The ratio of positive MCI-stained cells was as follows: for control, 2/100; for NFL, 23/100; and for WWE, 25/100 (chi-square test 19.7 with 2 degrees of freedom, $p<$ 0.0001). The ratio of positive PHF-stained cells was $2 / 100$ for control, 29/100 for NFL, and 32/100 for WWE groups (chi-square test 26 with 2 degrees of freedom, $\mathrm{p}<0.0001$ ). The ratio of positive CP-13-stained cells for control specimens was $2 / 100$, for NFL specimens it was $27 / 100$, and
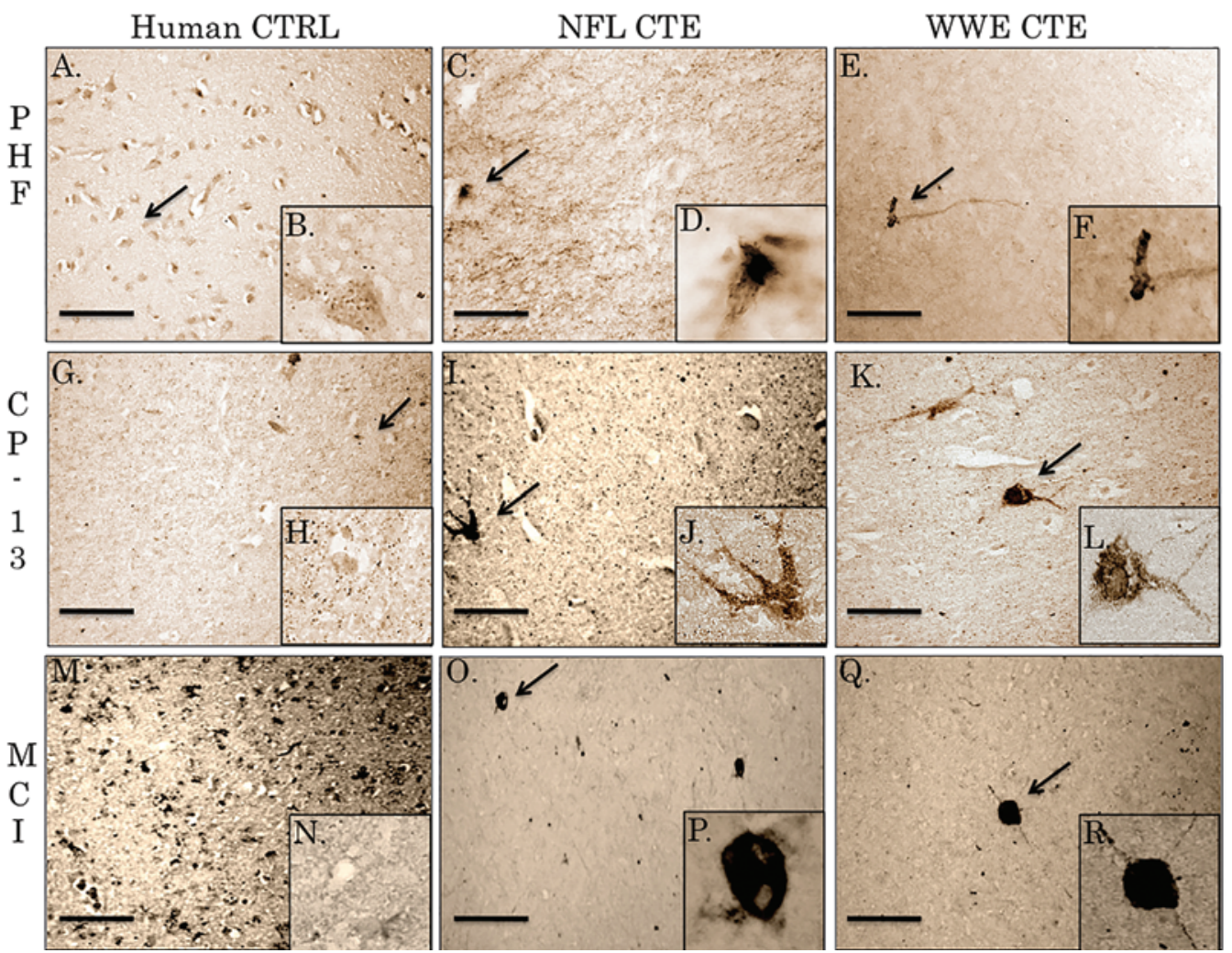

FIG. 4. Human CTE: characterized by distinct changes in tau hyperphosphorylation. The level of phosphorylation is dependent on the structural organization of tau and additionally on tau aggregation. PHFs are precursors to tau oligomers and indicate intrasomatic accumulation of tau. DAB staining revealed that PHFs were not present in the entorhinal cortex of an age-matched control (ratio 2/100) (A and B), but were present in the entorhinal cortices of an NFL player (ratio 29/100) (C and D) and a WWE wrestler (ratio 32/100) (E and F). CP-13 is a precursor for tau neurofibrillary tangles with a balanced 3-repeat to 4-repeat ratio. DAB staining revealed that CP-13 was not present in the entorhinal cortex of an age-matched control (ratio 2/100) (G and H) but was present in the entorhinal cortices of an NFL player (ratio 27/100) (I and J) and in a WWE wrestler (ratio 26/100) (K and L). $\mathrm{MCl}$ detects a distinct mutated version of tau that is present in neurodegenerative tauopathies. DAB staining revealed no $\mathrm{MCl}$ in the entorhinal cortex of an age-matched control (ratio 2/100) ( $\mathrm{M}$ and $\mathrm{N}$ ), but $\mathrm{MCl}$ was present in the entorhinal cortices of an NFL player (ratio 23/100) $(0$ and $P$ ) and in a WWE wrestler (ratio 25/100) (Q and R). Original magnification $\times 20$ and bar $=100 \mu \mathrm{m}(A, C, E, G, I, K$, $\mathrm{M}, \mathrm{O}$, and $\mathrm{Q})$; and original magnification $\times 63(\mathrm{~B}, \mathrm{D}, \mathrm{F}, \mathrm{H}, \mathrm{J}, \mathrm{L}, \mathrm{N}, \mathrm{P}$, and $\mathrm{R})$. Figure is available in color online only. 

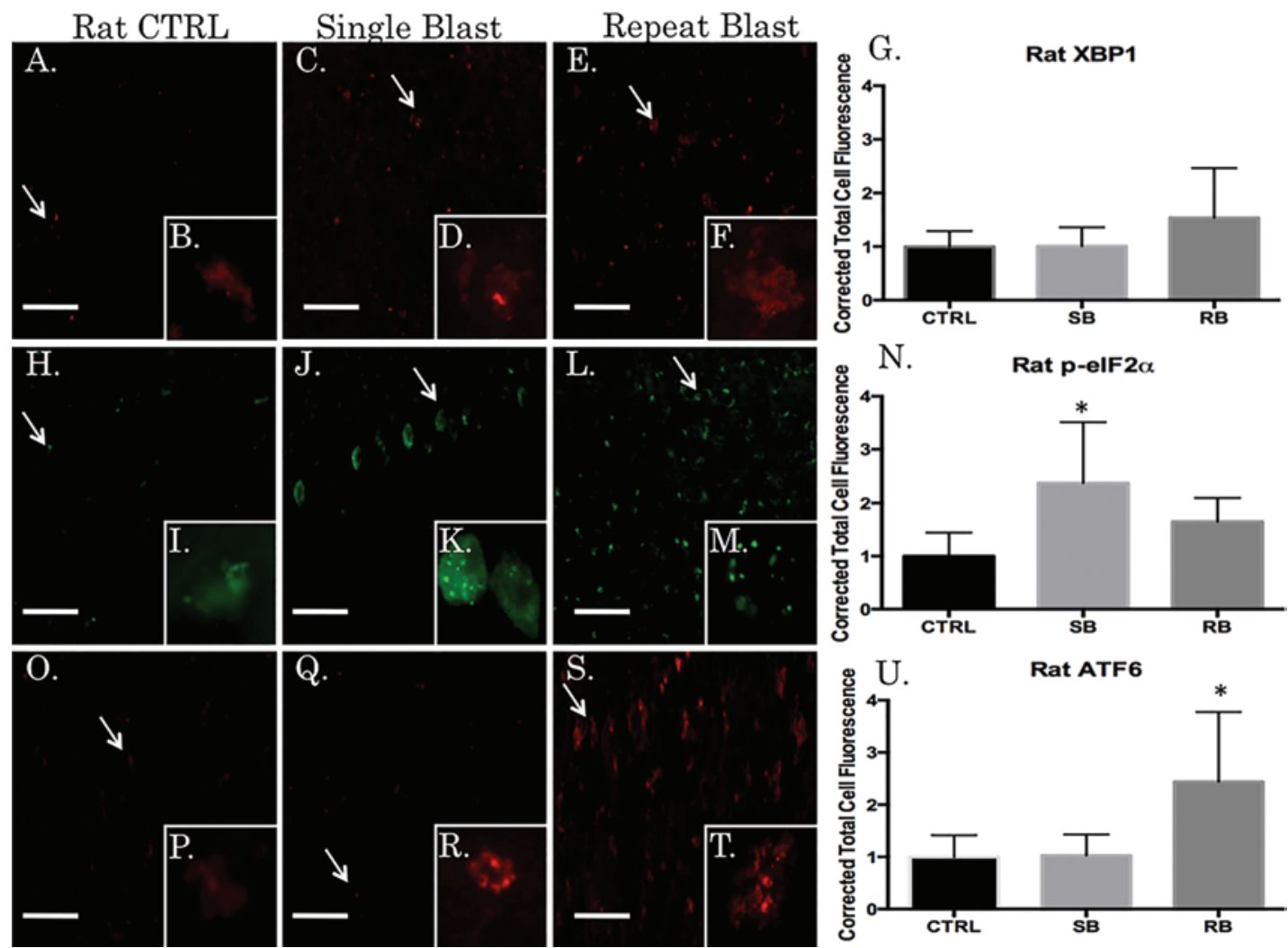

FIG. 5. ER stress as a defense mechanism. The ER stress response is a robust cellular mechanism that can help protect the cell from injury or toxic insult and restore homeostatic processes after injury. Arm one in particular provides vigorous neuroprotection after trauma. While acute increases in intracellular calcium trigger a modest ER stress response, the accumulation of unfolded proteins over time can enhance the vitality of the cellular ER response incorporating the detrimental Arms two and three. XBP1, Arm two of the ER stress response, was not significantly elevated in single-blast-exposed animals (C and D) or in repeat-blastexposed animals (E and F) compared with control (A and B), as depicted in the bar graph in G. P-elF2 $\alpha$, Arm one of the ER stress response, was significantly elevated in the single-blast-exposed animals $\left({ }^{*} p<0.05\right)(\mathrm{J}$ and $\mathrm{K}$ ) but not in the repeat-blast-exposed animals ( $L$ and $M$ ) compared with control $(\mathrm{H}$ and $\mathrm{I})$, as depicted in the bar graph in N. ATF6, Arm three of the ER stress response, was not significantly elevated in single-blast-exposed animals $(Q$ and $R)$ but was significantly elevated in repeat-blast-exposed animals ( $\left.{ }^{*} \mathrm{p}<0.05\right)$ (S and $\mathbf{T}$ ) compared with control $(\mathbf{O}$ and $\mathbf{P})$, as depicted in the bar graph in $\mathbf{U}$. Original magnification $\times 20$ and bar $=75 \mu \mathrm{m}(\mathrm{A}, \mathrm{C}, \mathrm{E}, \mathrm{H}, \mathrm{J}, \mathrm{L}, \mathrm{O}, \mathrm{Q}$, and S); and original magnification $\times 63(\mathrm{~B}, \mathrm{D}, \mathrm{F}, \mathrm{I}, \mathrm{K}, \mathrm{M}, \mathrm{P}, \mathrm{R}$, and $\mathrm{T})$. RB = repeat blast; $\mathrm{SB}=$ single blast. Figure is available in color online only.

for WWE specimens it was 26/100 (chi-square test 21.794 with 2 degrees of freedom, $p<0.0001)$. MCI, PHF, and $\mathrm{CP}-13$ mark a progression in tauopathy. $\mathrm{MCI}$ is an altered form of tau that appears after injury, whereas PHF (serine 396 and 404) is a precursor to tau oligomers, and CP-13 marks pretangle tau at serine 202.

\section{ER Stress Is Increased in Blast-Injured Animals and Human CTE}

The ER stress response is an important indicator for an injured cell with all three arms of the response-modulating cell survival. Acutely the ER stress response enhances survival, but chronic activation triggers apoptosis. ${ }^{15}$ The ER stress response has been implicated in both acute injuries, such as TBI, and chronic neurodegenerative disease development, such as Alzheimer's disease. ${ }^{17}$ We examined all three arms of the ER stress pathway with IHC both in our animals exposed to single- and repeat-blast injuries and in human CTE samples. Arm one, represented by p-eIF2 $\alpha$, was increased 24 hours after blast injury in the Sprague-Dawley rats $(\mathrm{F}(2,12)=4.07, \mathrm{p}<0.05)$ (Fig. $5 \mathrm{H}-\mathrm{N})$. Post hoc comparison showed statistical significance between control and a single-blast injury $(\mathrm{t}=2.852$, $\mathrm{p}<0.05)$. One-way ANOVA revealed no statistical significance for Arm two, represented by XBP1, 24 hours after a single- or repeat-blast injury in Sprague-Dawley rats (Fig. 5O-U). The 1-way ANOVA for Arm three, represented by ATF6, was statistically significant $(\mathrm{F}(2,12)=4.8, \mathrm{p}<0.05)$ for blast animals, and Bonferroni post hoc comparison revealed a significant difference between control and repeatblast groups $(t=2.710, p<0.05)($ Fig. $5 \mathrm{M}-\mathrm{R})$. p-eIF $2 \alpha$ was increased in the NFL and WWE CTE brains $(\mathrm{F}(2,12)$ $=13.08, \mathrm{p}<0.001)($ Fig. $6 \mathrm{H}-\mathrm{N})$. Post hoc comparison showed a significant difference between control and NFL brain samples $(\mathrm{t}=3.798, \mathrm{p}<0.01)$ and between control and WWE brain samples $(t=4.866, p<0.001)$. XBP1 was also increased in the CTE brains $(\mathrm{F}(2,12)=38.55, \mathrm{p}<0.001)$ (Fig. 6A-G). Post hoc comparison showed a significant difference between control and NFL brain tissue $(t=8.647$, 

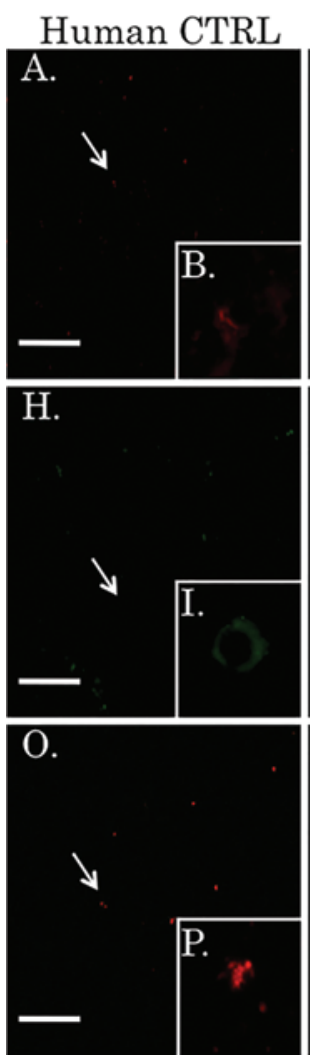
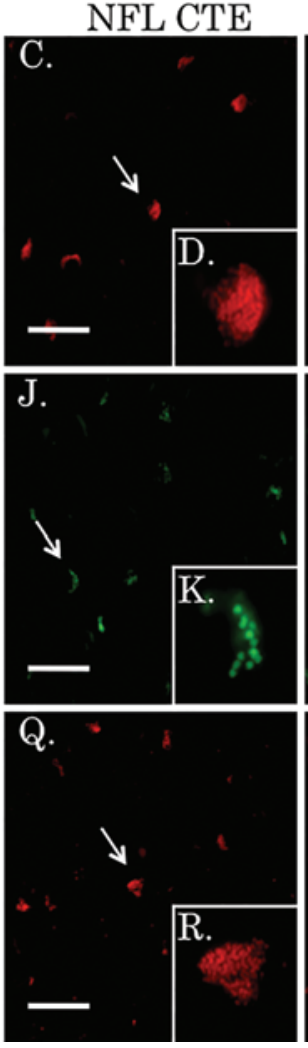
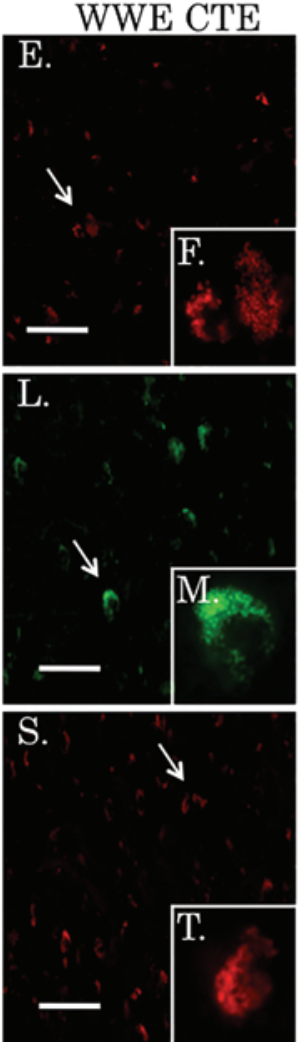
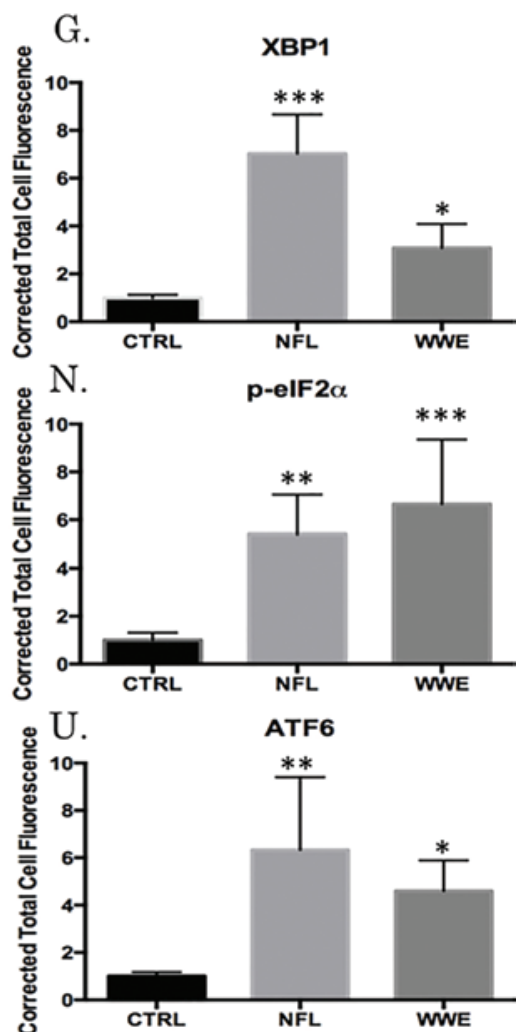

FIG. 6. Tauopathy implicated in the activation of the ER stress response. Since tau tangles are accumulations of aggregated protein, the cell recognizes an intracellular disturbance that triggers the release of calcium. The calcium rush is directly responsible for ER stress activation as well as initiating the ER stress response. All three arms of the ER stress cascade were increased in human CTE samples. XBP1, Arm two of the ER stress response, was significantly elevated in the NFL/CTE brain (** $p<0.001)$ $(C$ and $D)$ and in the WWE/CTE brain $\left({ }^{*} p<0.05\right)$ ( $E$ and $\left.F\right)$ compared with the control $(A$ and $B)$, as depicted in the bar graph in G. P-elF2 $\alpha$, Arm one of the ER stress response, was significantly elevated in the NFL/CTE brain $\left({ }^{* *} p<0.01\right)(\mathrm{J}$ and $\mathrm{K})$ and in the WWE/CTE brain $\left.{ }^{* * *} p<0.001\right)(\mathrm{L}$ and $\mathbf{M})$ compared with the control $(\mathbf{H}$ and I), as depicted in the bar graph in N. ATF6, Arm three of the ER stress response, was significantly elevated in the NFL/CTE brain $\left({ }^{* *} p<0.01\right)(Q$ and $R)$ and in the WWE/CTE brain $\left({ }^{*} p\right.$ $<0.05)$ (S and T) compared with control (O and P), as depicted in the bar graph in U. Original magnification $\times 20$ and bar $=75 \mu \mathrm{m}$ $(A, C, E, H, J, L, O, Q$, and S); and original magnification $\times 63(B, D, F, I, K, M, P, R$, and $T)$. Figure is available in color online only.

$\mathrm{p}<0.001)$ and between control and WWE brain tissue $(\mathrm{t}=$ $2.998, \mathrm{p}<0.05)$. Furthermore, ATF6 was increased in the CTE brains $(\mathrm{F}(2,12)=9.935, \mathrm{p}<0.01)$ (Fig. 6O-U). Post hoc comparison showed a significant difference between control and NFL brain samples $(\mathrm{t}=4.369, \mathrm{p}<0.01)$ and between control and WWE brain specimens $(t=2.951$, p $<0.05)$. CHOP was significantly elevated in human CTE brains $(\mathrm{F}(2,12)=24.17$, $\mathrm{p}<0.001)$ (Fig. 7A-G). Post hoc comparison showed a significant difference between control and NFL brain samples $(t=6.849 p<0.001)$. CHOP was also significantly elevated 24 hours after blast injury in Sprague-Dawley rats $(\mathrm{F}(2,12)=4.161, \mathrm{p}<0.05)$ (Fig. $7 \mathrm{H}-\mathrm{N})$. Post hoc comparison showed a significant difference between control and a single-blast injury $(t=2.875$, $\mathrm{p}<0.05)$.

\section{ER Stress Is Associated With Tau Hyperphosphorylation}

Tau hyperphosphorylation is an important precursor for the development of CTE. ${ }^{29}$ Currently, the mechanism of CTE development is not known, but ER stress offers to be a promising candidate based on prior work in neurotrauma showing an acute elevation of ER stress and chronic el- evation of ER stress in neurodegenerative disease such as Alzheimer's disease. We costained for ER stress activation, IRE1 $\alpha$, and tau hyperphosphorylation, AT270, in human CTE brains as well as Sprague-Dawley rats exposed to blast injury (Fig. 8). IRE1 $\alpha$ was used to represent the second arm of ER stress and has been colocalized with other tauopathies..$^{17}$ The Pearson's coefficient for human control brain was low $(r=0.173)$, but overlap coefficient was high for the NFL brain $(\mathrm{r}=0.881, \mathrm{k} 1=0.548$, and $\mathrm{k} 2$ $=1.416$ with threshold $\mathrm{A}=16$ and $\mathrm{B}=19)$ and the WWE brain $(\mathrm{r}=0.881, \mathrm{k} 1=0.615$, and $\mathrm{k} 2=1.261$ with threshold $\mathrm{A}=35$ and $\mathrm{B}=20$ ). Similarly, the Pearson's coefficient for the Sprague-Dawley control was low $(r=0.068)$, but overlap coefficient was high for single-blast $(\mathrm{r}=0.931$, $\mathrm{k} 1$ $=0.704$, and $\mathrm{k} 2=1.231$ with threshold $\mathrm{A}=16$ and $\mathrm{B}=14$ ) and repeat-blast $(\mathrm{r}=0.887, \mathrm{k} 1=0.518$, and $\mathrm{k} 2=1.518$ with threshold $\mathrm{A}=21$ and $\mathrm{B}=15$ ) injuries.

\section{Tau Kinase Activity and Hyperphosphorylation Increased With Repetitive Blast Injury}

GSK $3 \beta$ is activated by the ER stress response and contributes to chronic neurodegeneration. ${ }^{17}$ GSK3 $\beta$ is respon- 

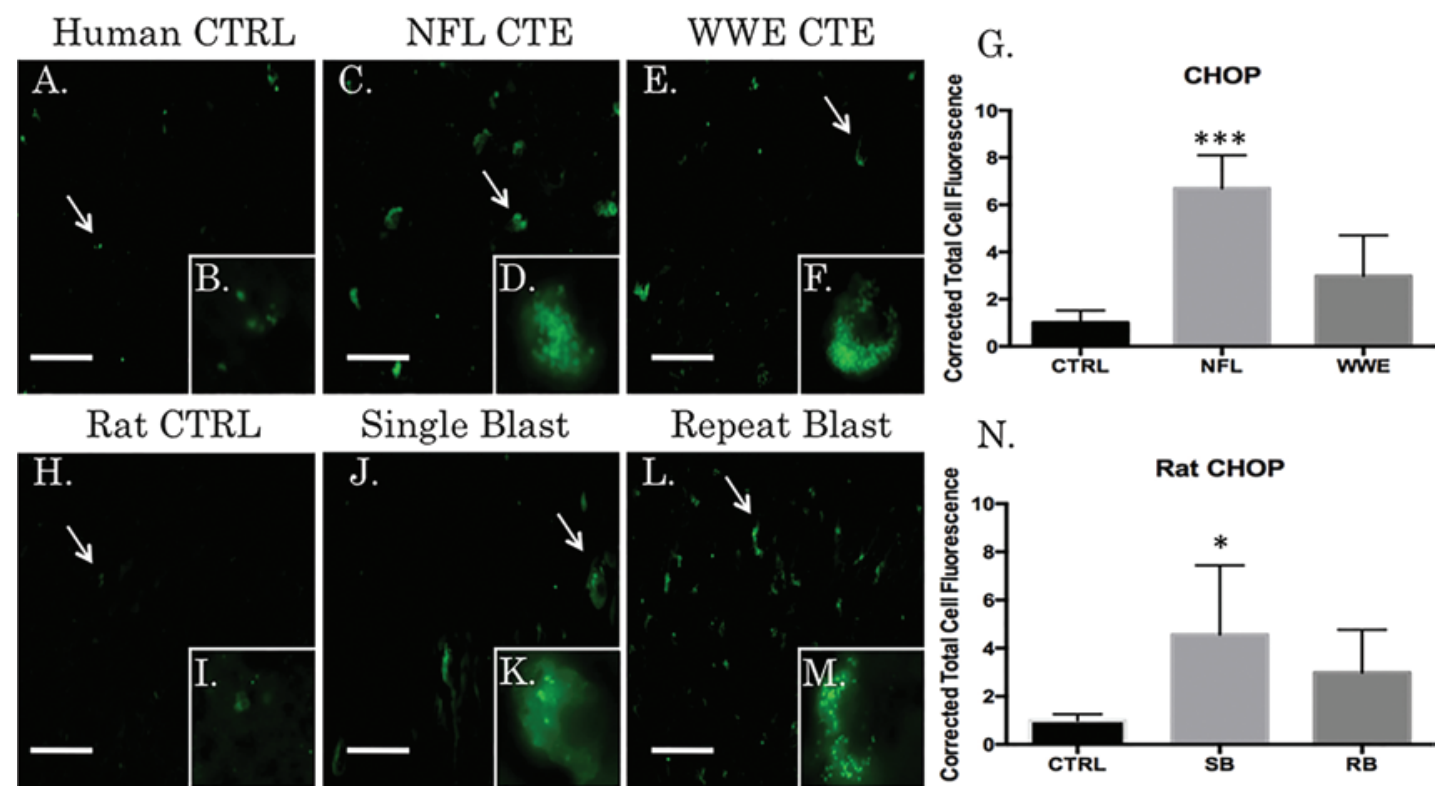

FIG. 7. CHOP is an important component of ER stress. A baseline level of $\mathrm{CHOP}$ is necessary in order for a cell to return to homeostasis. If $\mathrm{CHOP}$ is pushed above the threshold, apoptosis and tau hyperphosphorylation can occur. CHOP was significantly elevated in the NFL CTE brain (*** $<0.001)(C$ and $D)$ but not in the WWE CTE brain (E and F) compared with control (A and B), as depicted in the bar graph in G. CHOP was also significantly elevated in single-blast-exposed animals $\left({ }^{*} p<0.05\right)(\mathrm{J}$ and $\mathrm{K})$ but not in repeat-blast-exposed animals $(\mathrm{L}$ and $\mathrm{M})$ compared with control $(\mathrm{H}$ and $\mathrm{I})$ ), depicted in the bar graph in $\mathbf{N}$. Original magnification $\times 20$ and bar $=75 \mu \mathrm{m}(\mathrm{A}, \mathrm{C}, \mathrm{E}, \mathrm{H}, \mathrm{J}$, and $\mathrm{L})$; and original magnification $\times 63(\mathrm{~B}, \mathrm{D}, \mathrm{F}, \mathrm{I}, \mathrm{K}$, and $\mathrm{M})$. Figure is available in color online only.

sible for over one-third of all tau hyperphosphorylation and can lead to neuronal apoptosis. ${ }^{56}$ We propose that ER stress activates GSK3 $\beta$ in a biphasic manner both acutely and chronically. ${ }^{23} \mathrm{An}$ initial spike in activity is seen acutely that resolves, but over time GSK3 $\beta$ activity continually rises in conjunction with neurodegeneration. GSK3 $\beta$ was costained with a marker for tau hyperphosphorylation, AT100, in human CTE brains and Sprague-Dawley rats exposed to blast injury (Fig. 9). AT100 is a marker of tau hyperphosphorylation at threonine 212 and serine 214 , and GSK $3 \beta$ is known to phosphorylate at those sites.? Pearson's coefficient for human control brain was low $(\mathrm{r}=$ 0.286 ), but overlap coefficient was high for the NFL brain $(\mathrm{r}=0.846, \mathrm{k} 1=1.44$, and $\mathrm{k} 2=0.497$ with threshold $\mathrm{A}=$ 2 and $\mathrm{B}=2)$ and WWE brain $(\mathrm{r}=0.904, \mathrm{k} 1=2.316$, and $\mathrm{k} 2=0.353$ with threshold $\mathrm{A}=2$ and $\mathrm{B}=2$ ). The Pearson's coefficient for the Sprague-Dawley control was low $(\mathrm{r}=$ 0.374 ), but overlap coefficient was high for single-blast ( $\mathrm{r}$ $=0.934, \mathrm{k} 1=0.566$, and $\mathrm{k} 2=1.543$ with threshold $\mathrm{A}=6$ and $\mathrm{B}=6)$ and repeat-blast $(\mathrm{r}=0.807, \mathrm{k} 1=0.566$, and $\mathrm{k} 2$ $=1.543$ with threshold $\mathrm{A}=6$ and $\mathrm{B}=6$ ) injuries.

\section{Pharmacological Modulation of ER Stress Alters Tau Hyperphosphorylation}

Twenty-four hours postblast, we examined protein expression changes from Arms one and two of the ER stress response for single-blast-injured animals with and without DHA. A significant difference in GADD34 protein expression was seen $(F(3,12)=10.17, p<0.01)$. Post hoc analysis revealed a significant difference between control and single-blast groups $(t=6.009, p<0.01)$, between singleblast and single-blast+DHA groups $(\mathrm{t}=5.92, \mathrm{p}<0.01)$, and between single-blast and DHA-only groups $(\mathrm{t}=6.848, \mathrm{p}$ $<0.01$ ) (Fig. 10A). A significant difference in p-JNK was seen $(F(3,12)=9.779, p<0.01)$. Post hoc analysis revealed a significant difference between control and single-blast groups $(t=6.221, p<0.01)$, between single-blast and single-blast+DHA groups $(t=6.634, p<0.01)$, and between single-blast and DHA-only groups $(\mathrm{t}=6.04, \mathrm{p}<0.01)$ (Fig. 10B). In addition, we looked at BiP expression changes 3 weeks after repetitive blast exposure and how ER stress activation contributed to phosphorylation of tau kinases. A significant difference in $\mathrm{BiP}$ expression was seen $(\mathrm{F}(2,6)=$ $8.445, \mathrm{p}<0.05)$. Post hoc analysis revealed a significant difference between control and repeat-blast groups $(t=4.867$, $\mathrm{p}<0.05)$ and between repeat-blast and repeat-blast+DHA groups $(\mathrm{t}=5.185, \mathrm{p}<0.05)($ Fig. 11A). A significant difference in phosphorylation of GSK3 $\beta$ at tyrosine 216 was seen $(F(2,6)=31.73, p<0.001)$. Post hoc analysis revealed a significant difference between control and repeat-blast groups $(\mathrm{t}=10.31, \mathrm{p}<0.001)$ and between repeat-blast and repeat-blast+DHA groups $(t=9.085, \mathrm{p}<0.01)($ Fig. 11B) . All three arms of the ER stress response are known to increase CHOP. ${ }^{17} \mathrm{CHOP}$ is an important protein linking ER stress to neurodegeneration via the activation of tau kinases. A significant difference in $\mathrm{CHOP}$ protein expression between groups was seen 24 hours after blast exposure $(F(2,13)=9.8, p<0.01)$. Post hoc analysis revealed a significant difference between control and single-blast groups $(t=5.29, \mathrm{p}<0.05)$ and between single-blast and single-blast+DHA groups $(\mathrm{t}=5.361, \mathrm{p}<0.01)$. DHA reduced CHOP back toward control levels (Fig. 12A). AT270 is a marker of tau hyperphosphorylation at threonine 181 . The threonine 181 residue is commonly phosphorylated 


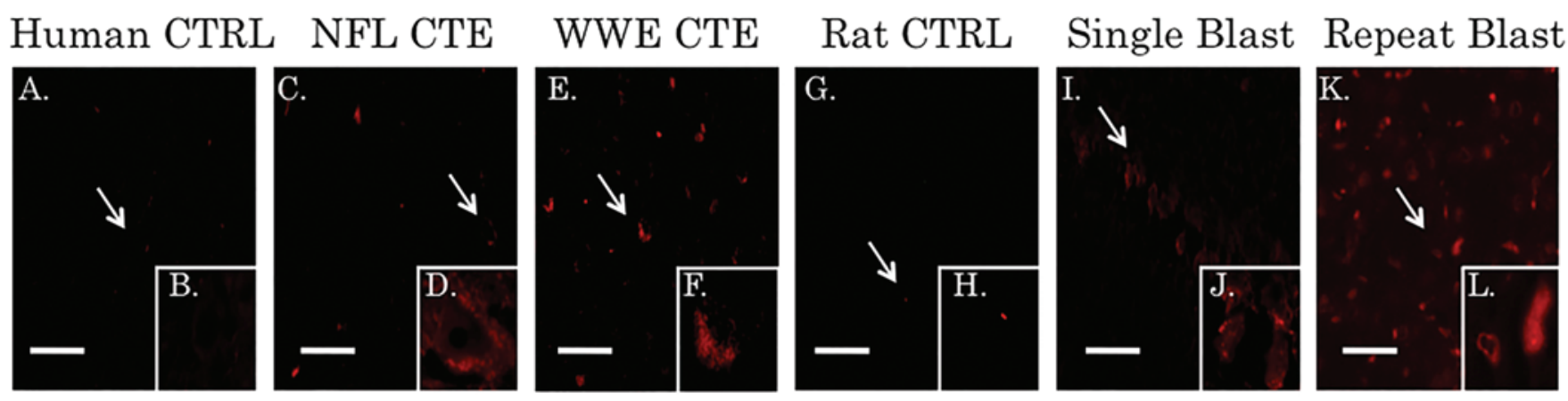

AT270
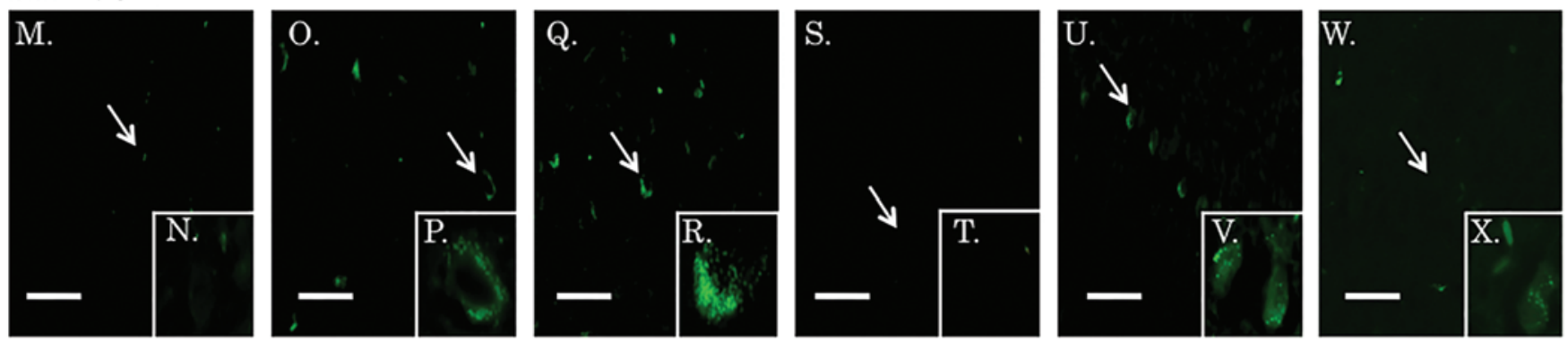

IRE1a
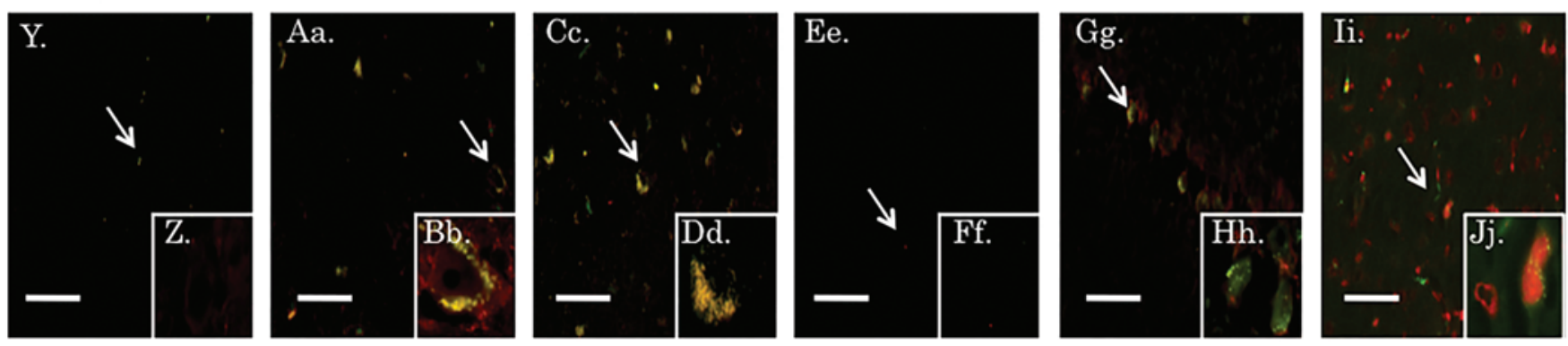

Overlay

FIG. 8. AT270 colocalized with IRE1 $\alpha$. AT270 is a marker for tau hyperphosphorylation at threonine 181. Tau hyperphosphorylation occurs after tau has dissociated from tubulin and is transported from the axon to the soma. What is unknown is if ER stress occurs in the same cells where tau hyperphosphorylation is present. We show that IRE1 $\alpha$, marker of Arm two of the ER stress response, has a strong overlap coefficient with AT270 for the NFL/CTE brain $(r=0.846)$ and WWE/CTE brain $(r=0.904)$ compared with weak Pearson's coefficient for the control $(r=0.286)$. The control has minimal AT270 (red) (A and B), IRE1 $\alpha$ (green) (M and N), and overlap (yellow) (Y and Z). The NFL/CTE brain has apparent AT270 (red) (C and D), IRE1 $\alpha$ (green) (O and P), and overlap (yellow) (Aa and Bb). The WWE/CTE brain has apparent AT270 (red) (E and F), IRE1 $\alpha$ (green) (Q and R), and overlap (yellow) (Cc and Dd). IRE1 $\alpha$ also has strong overlap coefficient with AT270 for single-blast-exposed animals $(r=0.934)$ and repeatblast-exposed animals $(r=0.807)$ compared with a weak Pearson's coefficient for the control $(r=0.374)$. The control has minimal AT270 (red) (G and H), IRE1 $\alpha$ (green) (S and T), and overlap (yellow) (Ee and Ff). The single-blast-exposed animal has apparent AT270 (red) (I and J), IRE1 $\alpha$ (green) (U and V), and overlap (yellow) ( $G g$ and Hh). The repeat-blast-exposed animal has apparent AT270 (red) (K and L), IRE1 $\alpha$ (green) (W and X), and overlap (yellow) (li and Jj). Original magnification $\times 20$ and bar $=75 \mu \mathrm{m}$ (A, C, E, G, I, K, M, O, Q, S, U, W, Y, Aa, Cc, Ee, Gg, and li); and original magnification ×63 (B, D, F, H, J, L, N, P, R, T, V, X, Z, Bb, $\mathrm{Dd}, \mathrm{Ff}, \mathrm{Hh}$, and Jj). Figure is available in color online only.

only when tau has translocated to the cell soma. ${ }^{20} \mathrm{~A}$ significant difference in AT270 between groups was seen 3 weeks after repeat-blast exposure $(F(2,9)=6.451, \mathrm{p}<0.05)$ (Fig. 12B). Post hoc analysis revealed a significant difference between control and repeat-blast groups $(t=4.81, p$ $<0.05)$ and between repeat-blast and repeat-blast+DHA groups $(\mathrm{t}=4.111, \mathrm{p}<0.05)$. CP-13 signals a change in tau hyperphosphorylation toward pretangle tau. A significant difference in CP-13 was seen 3 weeks after repeat-blast exposure $(\mathrm{F}(2,6)=13.23, \mathrm{p}<0.01)$ (Fig. 12C). Post hoc analysis revealed a significant difference between control and repeat-blast groups $(\mathrm{t}=5.778, \mathrm{p}<0.05)$ and between repeat-blast and repeat-blast+DHA groups $(\mathrm{t}=6.714, \mathrm{p}<$ $0.05)$.

\section{Repeat-Blast Exposure Induces CTE-Like Behavioral Changes Alleviated by DHA Administration}

CTE can lead to cognitive deficits and dementia. ${ }^{50} \mathrm{We}$ sought to reproduce CTE-like behavioral changes, in addition to the pathological changes described above, using a clinically relevant blast model. Using Morris water maze, a hippocampal-dependent cognitive performance test, we 


\section{Human CTRL NFL CTE WWE CTE Rat CTRL Single Blast Repeat Blast}
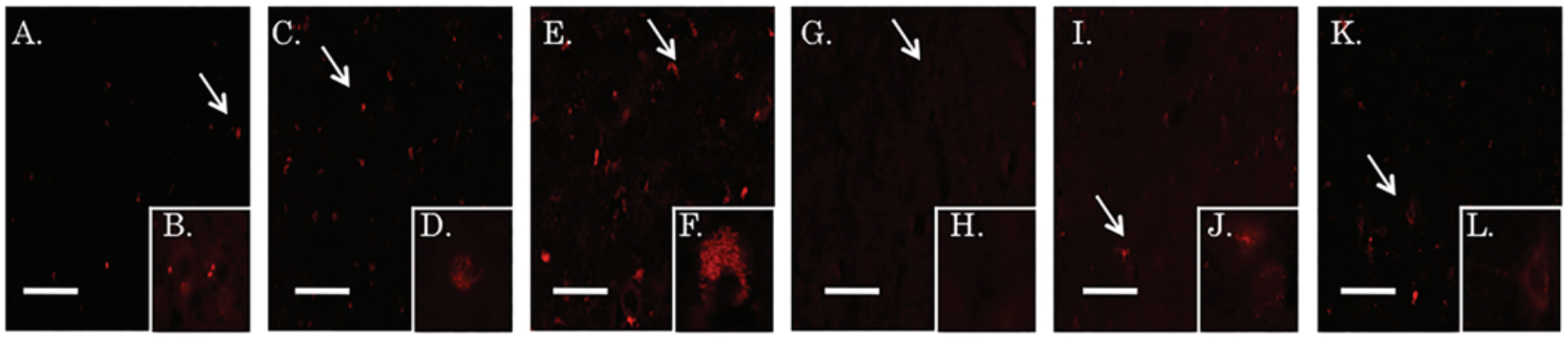

AT100
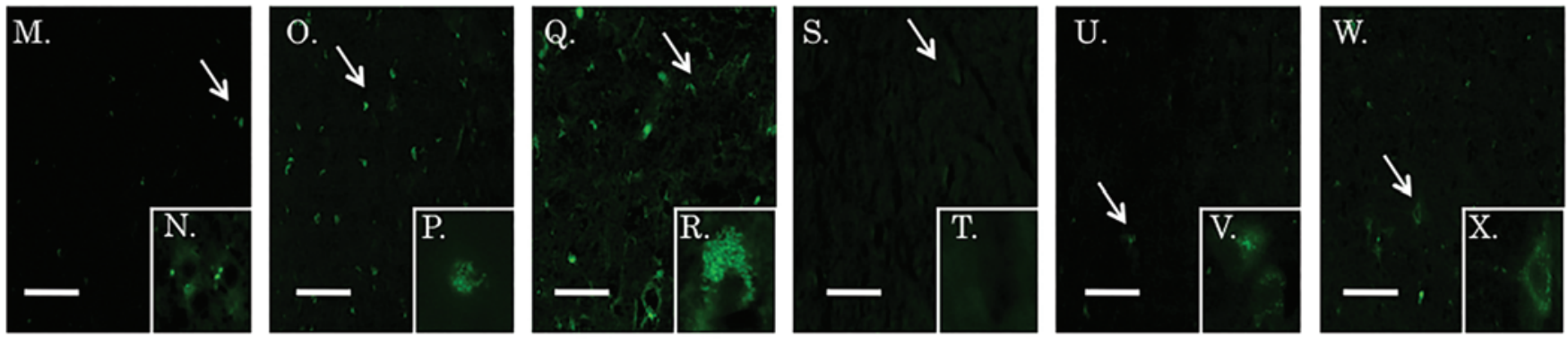

GSK3B
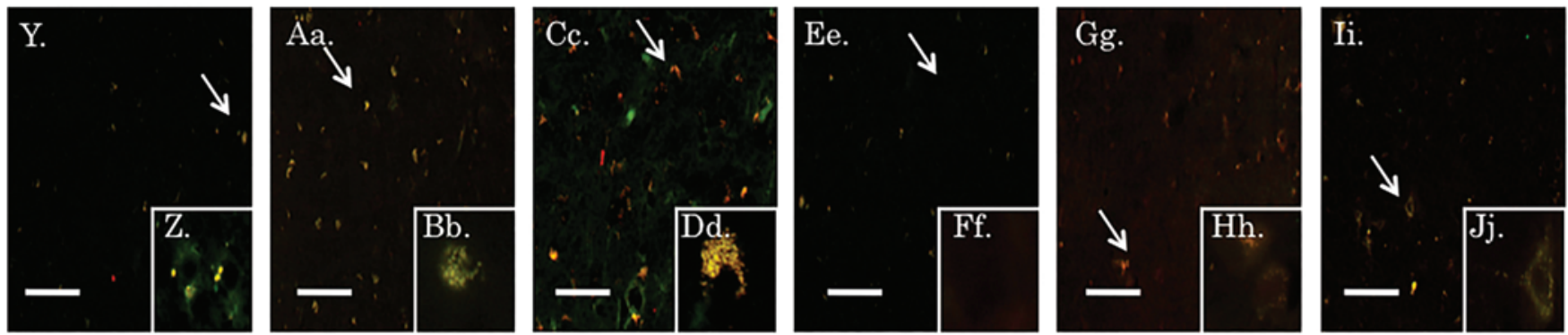

Overlay

FIG. 9. AT100 is a marker of tau hyperphosphorylation at threonine 212 and serine 214 . Tau is phosphorylated after injury by a select few kinases. GSK3 $\beta$ is by far the most important of these kinases, accounting for over one-third of all tau hyperphosphorylation. We show that GSK3 $\beta$ has a strong overlap coefficient with AT100 for the NFL/CTE brain $(r=0.881)$ and WWE brain $(r=0.881)$ compared with weak Pearson's coefficient for the control $(r=0.173)$. The control has minimal AT100 (red) $(A$ and B), GSK3 $\beta$ (green) (M and N), and overlap (yellow) (Y and Z). The NFL/CTE brain has apparent AT100 (red) (C and D), GSK3 $\beta$ (green) ( 0 and $\mathrm{P}$ ), and overlap (yellow) (Aa and Bb). The WWE/CTE brain has apparent AT100 (red) (E and F), GSK3 $\beta$ (green) ( $Q$ and $R$ ), and overlap (yellow) (Cc and Dd). GSK3 $\beta$ also has strong overlap coefficient with AT100 for single-blast-exposed animals $(r=0.931)$ and repeat-blast-exposed animals $(r=0.887)$ compared with a weak Pearson's coefficient for the control ( $r$ $=0.068$ ). The control has minimal AT100 (red) (G and H), GSK3 $\beta$ (green) (S and T), and overlap (yellow) (Ee and Ff). Singleblast-exposed animal has apparent AT100 (red) (I and J), GSK3 $\beta$ (green) (U and V), and overlap (yellow) (Gg and Hh). Repeatblast-exposed animal has apparent AT100 (red) (K and L), GSK3 $\beta$ (green) (W and X), and overlap (yellow) (li and Jj). Original magnification $\times 20$ and bar $=75 \mu \mathrm{m}(\mathrm{A}, \mathrm{C}, \mathrm{E}, \mathrm{G}, \mathrm{I}, \mathrm{K}, \mathrm{M}, \mathrm{O}, \mathrm{Q}, \mathrm{S}, \mathrm{U}, \mathrm{W}, \mathrm{Y}, \mathrm{Aa}, \mathrm{Cc}, \mathrm{Ee}, \mathrm{Gg}$, and li); and original magnification $\times 63(\mathrm{~B}$, $D, F, H, J, L, N, P, R, T, V, X, Z, B b, D d, F f, H h$, and Jj). Figure is available in color online only.

specifically evaluated postblast changes to determine potential deficits in memory acquisition and retention. ${ }^{37}$ In addition, we evaluated DHA, a proposed ER stress modulator, as a potential preventative/therapeutic agent. We found DHA administration 5 minutes following each blast and subsequent every-other-day administration attenuated learning deficits from repeat-blast exposure. A significant difference in learning across trial days $(\mathrm{F}(5,126)=19.68$, $\mathrm{p}$ $<0.001)$ and between groups $(\mathrm{F}(2,126)=38.53, \mathrm{p}<0.001)$ was observed. Post hoc analysis revealed a significant difference between control and repeat-blast groups for latency to platform on Day $2(t=3.633, p<0.01)$, Day $3(t=3.455$, $\mathrm{p}<0.01)$, Day $4(\mathrm{t}=4.057, \mathrm{p}<0.001)$, Day $5(\mathrm{t}=3.079, \mathrm{p}<$ $0.01)$, and Day $6(t=3.149, \mathrm{p}<0.01)$. A significant difference in latency to platform was also seen between repeatblast and repeat-blast+DHA groups on Day $2(\mathrm{t}=3.855, \mathrm{p}$ $<0.001)$, Day $3(\mathrm{t}=3.918, \mathrm{p}<0.001)$, Day $4(\mathrm{t}=4.415$, $\mathrm{p}<$ $0.001)$, Day $5(\mathrm{t}=3.442, \mathrm{p}<0.01)$, and Day $6(\mathrm{t}=3.283$, $\mathrm{p}<$ $0.01)$ (Fig. 12D). The probe trial revealed a significant difference between groups $(\mathrm{F}(2,21)=16.17, \mathrm{p}<0.001)$ in time exploring an area $300 \%$ of where the platform had been located prior (Fig. 12E). Post hoc comparison revealed 


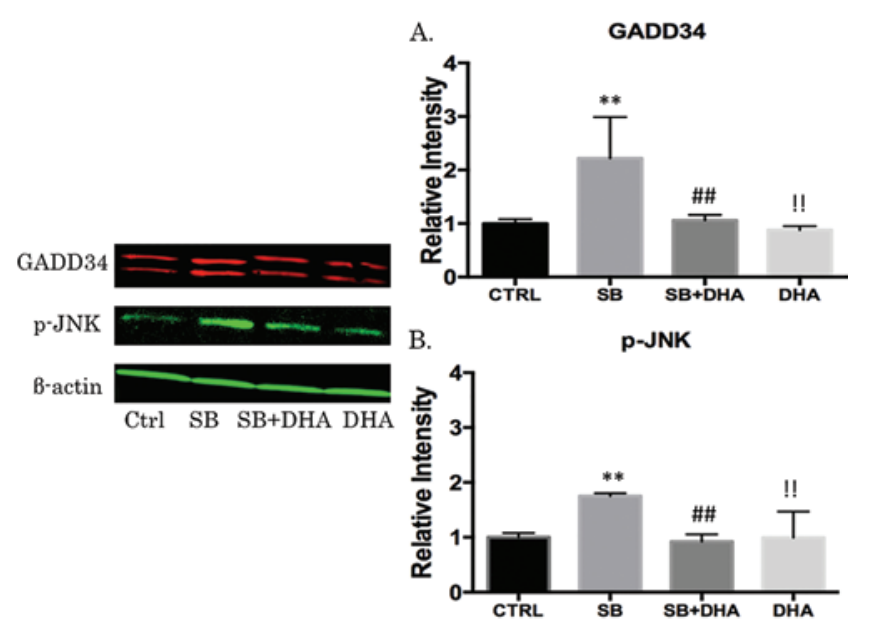

FIG. 10. DHA reduces acute ER stress markers. Arms one and two of the unfolded protein response are increased acutely after injury. GADD34 is a downstream marker of the first arm of the ER stress response and $p$-JNK is downstream of the second arm. A significant difference between groups for the acute markers was observed 24 hours after blast exposure. A: The single-blast group had increased expression of GADD34 compared with the control (**p<0.01), the $\mathrm{DHA}+$ single-blast group (\#\#p <0.01), and the DHA-alone group (!! $<<$ 0.01). B: The single-blast group had increased phosphorylation of JNK compared with the control $\left({ }^{* *} \mathrm{p}<0.01\right)$, the DHA+single-blast group (\#\#p $<0.01$ ), and the DHA-alone group (!! $<0.01)$. SB = single blast. Figure is available in color online only.

a significant difference between control and repeat-blast groups in time spent exploring an area $300 \%$ of where the platform had been $(t=7.331, \mathrm{p}<0.001)$ as well as between repeat-blast and DHA+repeat-blast groups $(\mathrm{t}=6.532, \mathrm{p}<$ $0.001)$.

\section{Discussion}

ER stress has recently been implicated in chronic neurodegeneration. ${ }^{31}$ Tauopathies are the result of aggregated and mutated proteins that cause immunoexcitotoxicity and ER stress activation. ${ }^{5}$ The ER responds to toxic buildup of proteins by shutting down protein synthesis and inhibiting protein folding. ${ }^{42}$ The ER stress response may accelerate tauopathy by facilitating tau hyperphosphorylation and rapid tau aggregation that leads to neurofibrillary tangles. ${ }^{17} \mathrm{CTE}$ is unique among tauopathies in that its onset and progression are thought to be associated with repetitive head injury. ${ }^{2,25}$ Currently, diagnostic and treatment options are unavailable for this disease, although they are improving with the use of the $\left[{ }^{18} \mathrm{~F}\right] \mathrm{FDDNP}$-PET imaging probe ${ }^{46}$ The growing number of athletes and soldiers experiencing CTE, characterized by symptoms of impulsivity, cognitive decline, and depression, urges renewed focus on understanding disease mechanism and disease progression. ${ }^{53}$ It has long been postulated that TBI leads to chronic neurodegeneration..$^{30,32,47}$ Recent findings have confirmed that changes in tau, amyloid, and cognition can result from neurotrauma. ${ }^{9,34,51}$ The mechanisms underlying how these changes develop is currently unknown.

We show for the first time that ER stress is intimately associated with CTE in human clinical postmortem specimens and in the development of CTE-like pathology and

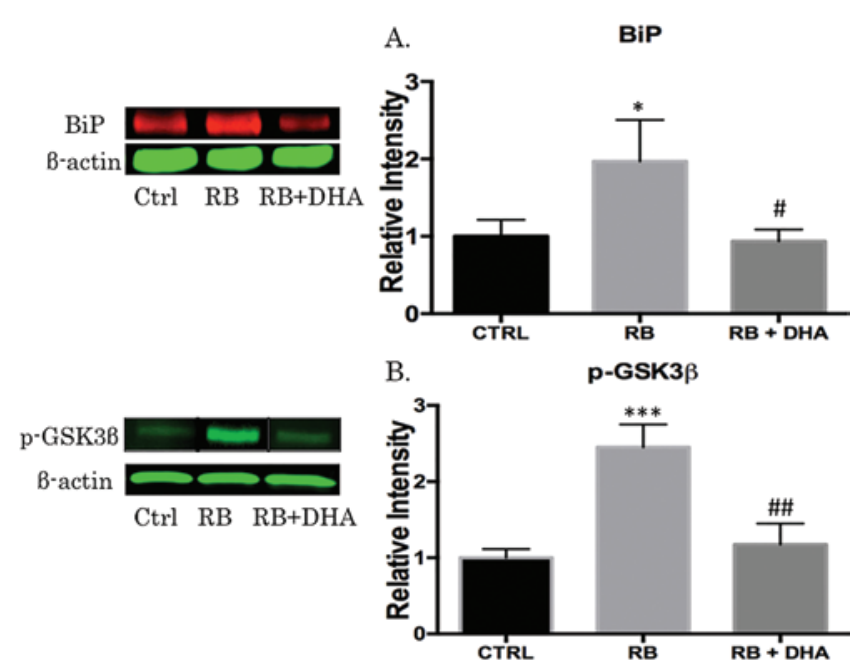

FIG. 11. ER stress plays an important role in activation of tau kinases. Tau hyperphosphorylation feeds back and activates the ER stress response by causing the dissociation of BiP from the ER membrane. A significant difference between groups was observed 3 weeks after repetitive blast exposure for BiP. A: The repeat-blast group had increased levels of BiP compared with the control $\left({ }^{*} p<0.05\right)$ and with the repeatblast+DHA group $(\# p<0.05)$. Activation of the tau kinase GSK3 $\beta$ can lead to increased tau pathology following TBI. A significant difference between groups was reported for p-GSK3 $\beta$. B: The repeat-blast group has increased phosphorylation of GSK3 $\beta$ at tyrosine 216 compared with the control $\left({ }^{* *} p<0.001\right)$ and with the repeat-blast+DHA group $(\# \# p<$ $0.01) . R B=$ repeat blast. Figure is available in color online only.

behavioral deficits in an animal model of repetitive neurotrauma. ER stress is acutely triggered by rapid changes in intracellular calcium following brain injury. ${ }^{19}$ All three arms of the ER stress response were significantly elevated in human CTE brains, indicating that not only does neurotrauma lead to acute increases in ER stress, but also tau pathology likely contributes to persistent elevations in ER stress during chronic neurodegeneration. $\mathrm{CHOP}$, a downstream target of ER stress, was increased in the NFL/CTE brain, but not in the WWE/CTE brain. The WWE/CTE case had more severe neurofibrillary tangle pathology, whereas the process of tau hyperphosphorylation was ongoing in the NFL CTE brain. Therefore, CHOP may be associated with active changes in the tau phosphorylation state. Begum and colleagues have shown that p-eIF $2 \alpha$ is increased 24-48 hours after TBI, but downstream components of Arm two of the ER stress response were not increased until 3-7 days after injury. ${ }^{4}$ These findings likely account for why we did not observe a statistically significant increase in XBP1 following single- and repeat-blast exposure at 24 hours after injury. The other branch of Arm two, p-JNK, however, was increased at 24 hours, but the expression was reduced when DHA was given. ATF6 was significantly increased 24 hours following repeat blast, indicating an appropriate cleavage from the membrane due to an accumulation of unfolded proteins. ${ }^{57}$ Future work will examine the effects of repeat blasts on ER stress activation at subacute (3-7 day) time points. The CHOP increase was not statistically significant for repeat-blast when compared with control groups 24 hours postinjury. It is likely that $\mathrm{CHOP}$ will be increased after repeat blasts 
A.
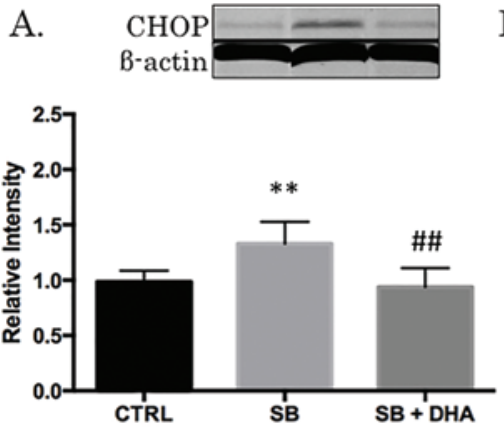

B.

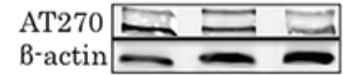

C.

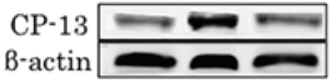

D.

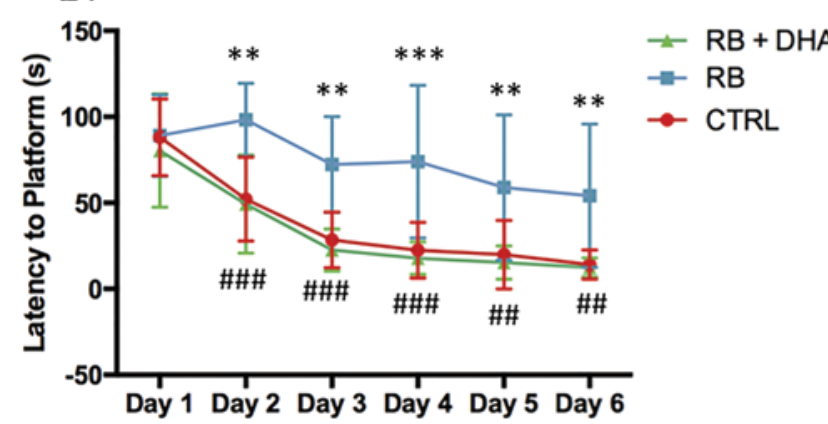

E.

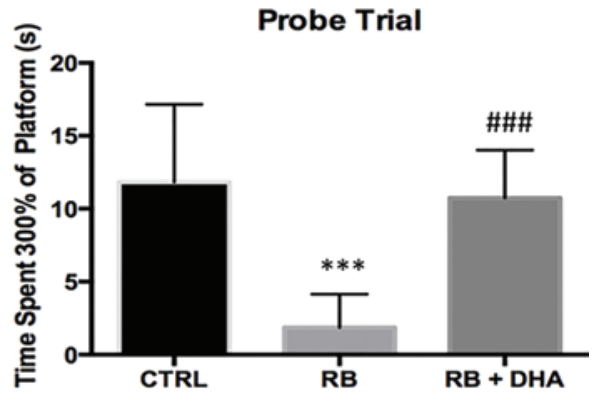

FIG. 12. DHA decreases ER stress and tau while preventing cognitive decline. CHOP is a key downstream target of all three arms of the ER stress pathway. Among its many roles, CHOP contributes to apoptosis, activation of tau kinases, and disruption of the mitochondria. A significant difference between groups was observed 24 hours after blast exposure. A: The single-blast group had a significant increase in $\mathrm{CHOP}\left({ }^{* *} \mathrm{p}<0.01\right)$ compared with the control. The DHA+blast group had a significant reduction in $\mathrm{CHOP}$ $(\# \# p<0.01)$ compared with the single-blast group only. AT270 is a marker of tau hyperphosphorylation that symbolizes the transition phase for tauopathy development. B: Repeat blast increased AT270 compared with the control ( $\left.{ }^{*} p<0.05\right)$. DHA+repeat blast significantly reduced AT270 (\#p < 0.05) compared with a repeat blast alone. C: Repeat blast increased CP-13 compared with the control ( $\left.{ }^{*} p<0.05\right)$. DHA+repeat blast significantly reduced CP-13 (\#\#p<0.05) compared with repeat blast only. To establish the animals' cognitive performance and the ability to learn, they were tested in a Morris water maze. See the Morris Water Maze section for a description of the protocol used for this experiment. D: A significant difference in learning across trial days $(p<0.001)$ and between groups $(p<0.001)$ was observed. A significant difference between control and repeat-blast groups for latency to platform was observed on Day $2\left(^{* * t}=3.633, p<0.01\right)$, Day $3\left({ }^{* * t} t=3.455, p<0.01\right)$, Day $4\left({ }^{* *} t=4.057, p<0.001\right)$, Day $5\left({ }^{* * t}=3.079\right.$, $p<0.01)$, and Day $6\left({ }^{* *} t=3.149, p<0.01\right)$. A significant difference in latency to platform was also seen between repeat-blast and repeat-blast+DHA groups on Day $2(\# \# t=3.855, p<0.001)$, Day $3(\# \# t=3.918, p<0.001)$, Day $4(\# \#$ t $=4.415, p<0.001)$, Day $5(\# \# \mathrm{t}=3.442, p<0.01)$, and Day $6(\# \# \mathrm{t}=3.283, p<0.01)$. $\mathrm{E}$ : A significant difference between control and repeat-blast groups $\left({ }^{* * *} p<0.001\right)$ and between repeat-blast and repeat-blast+DHA groups $(\# \# p<0.001)$ was seen on probe trial. Figure is available in color online only.

starting 3 days postinjury. The initial lack of change is likely due to inflammatory preconditioning from the short interval between blast exposures.

Using our clinically relevant and validated blast model, we examined active changes in tau following blast-induced TBI and correlated these changes with human CTE pathology. The ER stress-activated tau kinase, GSK3 $\beta$, was significantly increased after repetitive blast injury, and subsequent tau hyperphosphorylation was colocalized within the same cells where ER stress activation was occurring. The activating phosphorylation of GSK $3 \beta$ at tyrosine 216 was reduced by DHA administration. Future work will focus on teasing out exactly how an increase in CHOP may activate GSK $3 \beta$ and in which specific cell types these changes are occurring. Interestingly, ATF6 from Arm three of the ER stress pathway has been shown to significantly elevate Akt. ${ }^{59}$ Since GSK $3 \beta$ triggers apoptosis, Akt is necessary to directly inhibit GSK3 $\beta$ and promote cell survival. Neuronal survival is required for transsynaptic tau propagation. ${ }^{40}$ Akt is a well-known tau kinase that fosters the transsynaptic spread of tau tangles over time. ${ }^{44}$ We plan to investigate these interactions between Akt and GSK3 $\beta$ in follow-up studies. It is likely that ATF6 is continually activated at extended time points in the injury cascade compared with Arms one and two of the ER stress response, which would account for the initial increase we observed in GSK3 $\beta$ following blast exposure.

Many of the experimental drugs that target the ER stress response have a high affinity for a single arm of the pathway at upstream targets. While beneficial acutely, these compounds fail to address the compensatory response of the remaining arms of the ER stress response and subsequent crosstalk between the arms. The crosstalk is what allows CHOP to be pushed above threshold and apoptosis and tau hyperphosphorylation to ensue. ${ }^{15}$ The ER stress response is a cellular defense mechanism. If protein folding cannot be restored quickly, the ER triggers the cell to undergo apoptosis. Too much activation of 
any arm of the ER stress response can accelerate this process and contribute to chronic neurodegeneration. ${ }^{12}$ DHA acutely inhibits all three arms of the ER stress response after injury by limiting the intracellular calcium surge. ${ }^{3}$ DHA reduced GADD34 and p-JNK acutely, but also decreased BiP 3 weeks following repeat blasts. By acting at a target step above ER stress activation and BiP dissociation, DHA prevents the compensatory reverberation from other arms of the ER stress pathway, allowing little to no acute protein regulation resulting from injury. In addition, DHA has recently been shown to readily enter the brain and inhibit neurofibrillary tangle formation in a murine model of Alzheimer's disease..$^{13,22}$ Linking these two important properties of DHA is a topic of ongoing investigation.

\section{Conclusions}

Markers of ER stress were increased in both SpragueDawley rat samples and human CTE specimens. These markers were colocalized with tau pathology in hippocampal neurons. Sprague-Dawley rats showed pretangle tau changes comparable to those of human CTE 3 weeks after a repetitive blast. Most importantly, we showed that the omega-3 derivative, DHA, reduced ER stress-mediated CHOP expression, GADD34 expression, and p-JNK at 24 hours and additionally reduced tau hyperphosphorylation, BiP dissociation, and phosphorylation of GSK3 $\beta$ at 3 weeks postinjury. DHA also decreased cognitive decline measured by Morris water maze when given 5 minutes after each blast exposure. DHA appears to have pharmacotherapeutic potential as a preventative treatment against the development of CTE and may be a promising treatment for various other forms of neurotrauma. ${ }^{14}$

\section{Acknowledgments}

We would like to acknowledge the work done by Nic Jordan, BS, and Kelly Smith, BS, for assistance with IHC. We would also like to thank Dr. James O'Callaghan of CDC/NIOSH for use of resources for Western blot quantification, and Diana Richardson of CDC/NIOSH for tissue preparation for IHC. We also thank Dr. Rae Matsumoto for use of Western blot resources in her laboratory, and the West Virginia University Microscopy Imaging Facility for assistance with microscopy. We additionally thank James E. Robson and Peter Bennett for construction of the blast model and Dr. Robert Gettens and Nic St. John for design of the model. We thank Dr. Peter Davies for his kind gift of PHF, CP-13, and MCI antibodies.

A Research Funding and Development Grant from the West Virginia University Health Sciences Center Office of Research and Graduate Education supported this work along with the West Virginia University Department of Neurosurgery. Drs. Turner and Robson were supported by NIH training grants (GM08174 and NS007491). Brandon Lucke-Wold was supported by an American Foundation of Pharmaceutical Education Predoctoral Grant, an American Medical Association Foundation Seed Grant, and a Neurosurgery Research and Education Foundation Medical Student Summer Research Fellowship.

\section{References}

1. Abisambra JF, Jinwal UK, Blair LJ, O’Leary JC III, Li Q, Brady $S$, et al: Tau accumulation activates the unfolded protein response by impairing endoplasmic reticulum-associated degradation. J Neurosci 33:9498-9507, 2013
2. Aungst SL, Kabadi SV, Thompson SM, Stoica BA, Faden AI: Repeated mild traumatic brain injury causes chronic neuroinflammation, changes in hippocampal synaptic plasticity, and associated cognitive deficits. J Cereb Blood Flow Metab 34:1223-1232, 2014

3. Begum G, Harvey L, Dixon CE, Sun D: ER stress and effects of DHA as an ER stress inhibitor. Transl Stroke Res 4:635-642, 2013

4. Begum G, Yan HQ, Li L, Singh A, Dixon CE, Sun D: Docosahexaenoic acid reduces ER stress and abnormal protein accumulation and improves neuronal function following traumatic brain injury. J Neurosci 34:3743-3755, 2014

5. Blaylock RL, Maroon J: Immunoexcitotoxicity as a central mechanism in chronic traumatic encephalopathy-A unifying hypothesis. Surg Neurol Int 2:107, 2011

6. Bolte S, Cordelières FP: A guided tour into subcellular colocalization analysis in light microscopy. J Microsc 224:213232, 2006

7. Cavallini A, Brewerton S, Bell A, Sargent S, Glover S, Hardy $\mathrm{C}$, et al: An unbiased approach to identifying tau kinases that phosphorylate tau at sites associated with Alzheimer disease. J Biol Chem 288:23331-23347, 2013

8. Chen CH, Shaikenov T, Peterson TR, Aimbetov R, Bissenbaev AK, Lee SW, et al: ER stress inhibits mTORC2 and Akt signaling through GSK-3 $\beta$-mediated phosphorylation of rictor. Sci Signal 4:ra10, 2011

9. Dapul HR, Park J, Zhang J, Lee C, DanEshmand A, Lok J, et al: Concussive injury before or after controlled cortical impact exacerbates histopathology and functional outcome in a mixed traumatic brain injury model in mice. $\mathbf{J}$ Neurotrauma 30:382-391, 2013

10. Dinapoli VA, Benkovic SA, Li X, Kelly KA, Miller DB, Rosen CL, et al: Age exaggerates proinflammatory cytokine signaling and truncates signal transducers and activators of transcription 3 signaling following ischemic stroke in the rat. Neuroscience 170:633-644, 2010

11. Farook JM, Shields J, Tawfik A, Markand S, Sen T, Smith $\mathrm{SB}$, et al: GADD34 induces cell death through inactivation of Akt following traumatic brain injury. Cell Death Dis 4:e754, 2013

12. Fonseca AC, Ferreiro E, Oliveira CR, Cardoso SM, Pereira $\mathrm{CF}$ : Activation of the endoplasmic reticulum stress response by the amyloid-beta 1-40 peptide in brain endothelial cells. Biochim Biophys Acta 1832:2191-2203, 2013

13. Freund Levi Y, Vedin I, Cederholm T, Basun H, Faxén Irving $\mathrm{G}$, Eriksdotter M, et al: Transfer of omega-3 fatty acids across the blood-brain barrier after dietary supplementation with a docosahexaenoic acid-rich omega-3 fatty acid preparation in patients with Alzheimer's disease: the OmegAD study. J Intern Med 275:428-436, 2014

14. Hasadsri L, Wang BH, Lee JV, Erdman JW, Llano DA, Barbey AK, et al: Omega-3 fatty acids as a putative treatment for traumatic brain injury. J Neurotrauma 30:897-906, 2013

15. Hetz C, Mollereau B: Disturbance of endoplasmic reticulum proteostasis in neurodegenerative diseases. Nat Rev Neurosci 15:233-249, 2014

16. Hiramatsu N, Messah C, Han J, LaVail MM, Kaufman RJ, Lin JH: Translational and posttranslational regulation of XIAP by eIF2 $\alpha$ and ATF4 promotes ER stress-induced cell death during the unfolded protein response. Mol Biol Cell 25:1411-1420, 2014

17. Hoozemans JJ, Scheper W: Endoplasmic reticulum: the unfolded protein response is tangled in neurodegeneration. Int J Biochem Cell Biol 44:1295-1298, 2012

18. Kang EB, Kwon IS, Koo JH, Kim EJ, Kim CH, Lee J, et al: Treadmill exercise represses neuronal cell death and inflammation during $A \beta$-induced ER stress by regulating unfolded protein response in aged presenilin 2 mutant mice. Apoptosis 18:1332-1347, 2013 
19. Kaufman RJ, Malhotra JD: Calcium trafficking integrates endoplasmic reticulum function with mitochondrial bioenergetics. Biochim Biophys Acta 1843:2233-2239, 2014

20. Kester MI, Goos JD, Teunissen CE, Benedictus MR, Bouwman FH, Wattjes MP, et al: Associations between cerebral small-vessel disease and Alzheimer disease pathology as measured by cerebrospinal fluid biomarkers. JAMA Neurol 71:855-862, 2014

21. Larner SF, Hayes RL, McKinsey DM, Pike BR, Wang KK: Increased expression and processing of caspase-12 after traumatic brain injury in rats. J Neurochem 88:78-90, 2004

22. Lebbadi M, Julien C, Phivilay A, Tremblay C, Emond V, Kang JX, et al: Endogenous conversion of omega- 6 into omega-3 fatty acids improves neuropathology in an animal model of Alzheimer's disease. J Alzheimers Dis 27:853-869, 2011

23. Liu ZC, Fu ZQ, Song J, Zhang JY, Wei YP, Chu J, et al: Bip enhanced the association of GSK-3 $\beta$ with tau during ER stress both in vivo and in vitro. J Alzheimers Dis 29:727740,2012

24. Lucke-Wold BP, Turner RC, Logsdon AF, Bailes JE, Huber JD, Rosen CL: Linking traumatic brain injury to chronic traumatic encephalopathy: identification of potential mechanisms leading to neurofibrillary tangle development. J Neurotrauma 31:1129-1138, 2014

25. MacDonald CL, Johnson AM, Nelson EC, Werner NJ, Fang R, Flaherty SF, et al: Functional status after blast-plus-impact complex concussive traumatic brain injury in evacuated United States military personnel. J Neurotrauma 31:889-898, 2014

26. McKee AC, Daneshvar DH, Alvarez VE, Stein TD: The neuropathology of sport. Acta Neuropathol 127:29-51, 2014

27. McKee AC, Gavett BE, Stern RA, Nowinski CJ, Cantu RC, Kowall NW, et al: TDP-43 proteinopathy and motor neuron disease in chronic traumatic encephalopathy. J Neuropathol Exp Neurol 69:918-929, 2010

28. McKee AC, Stern RA, Nowinski CJ, Stein TD, Alvarez VE, Daneshvar DH, et al: The spectrum of disease in chronic traumatic encephalopathy. Brain 136:43-64, 2013

29. Mez J, Stern RA, McKee AC: Chronic traumatic encephalopathy: where are we and where are we going? Curr Neurol Neurosci Rep 13:407, 2013

30. Miyauchi T, Wei EP, Povlishock JT: Evidence for the therapeutic efficacy of either mild hypothermia or oxygen radical scavengers after repetitive mild traumatic brain injury. $\mathbf{J}$ Neurotrauma 31:773-781, 2014

31. Moreno JA, Halliday M, Molloy C, Radford H, Verity N, Axten JM, et al: Oral treatment targeting the unfolded protein response prevents neurodegeneration and clinical disease in prion-infected mice. Sci Transl Med 5:206ra138, 2013

32. Nakagawa A, Manley GT, Gean AD, Ohtani K, Armonda R, Tsukamoto A, et al: Mechanisms of primary blast-induced traumatic brain injury: insights from shock-wave research. J Neurotrauma 28:1101-1119, 2011

33. Noble JM, Hesdorffer DC: Sport-related concussions: a review of epidemiology, challenges in diagnosis, and potential risk factors. Neuropsychol Rev 23:273-284, 2013

34. Omalu B, Bailes J, Hamilton RL, Kamboh MI, Hammers $\mathrm{J}$, Case M, et al: Emerging histomorphologic phenotypes of chronic traumatic encephalopathy in American athletes. Neurosurgery 69:173-183, 2011

35. Omalu BI, Bailes J, Hammers JL, Fitzsimmons RP: Chronic traumatic encephalopathy, suicides and parasuicides in professional American athletes: the role of the forensic pathologist. Am J Forensic Med Pathol 31:130-132, 2010

36. Omalu BI, Fitzsimmons RP, Hammers J, Bailes J: Chronic traumatic encephalopathy in a professional American wrestler. J Forensic Nurs 6:130-136, 2010

37. Petraglia AL, Plog BA, Dayawansa S, Chen M, Dashnaw ML, Czerniecka K, et al: The spectrum of neurobehavioral sequelae after repetitive mild traumatic brain injury: a novel mouse model of chronic traumatic encephalopathy. J Neurotrauma 31:1211-1224, 2014

38. Placido AI, Oliveira CR, Moreira PI, Pereira CM: Enhanced amyloidogenic processing of amyloid precursor protein and cell death under prolonged endoplasmic reticulum stress in brain endothelial cells. Mol Neurobiol 51:571-590, 2015

39. Plattner F, Angelo M, Giese KP: The roles of cyclin-dependent kinase 5 and glycogen synthase kinase 3 in tau hyperphosphorylation. J Biol Chem 281:25457-25465, 2006

40. Pooler AM, Phillips EC, Lau DH, Noble W, Hanger DP: Physiological release of endogenous tau is stimulated by neuronal activity. EMBO Rep 14:389-394, 2013

41. Robson MJ, Turner RC, Naser ZJ, McCurdy CR, O'Callaghan JP, Huber JD, et al: SN79, a sigma receptor antagonist, attenuates methamphetamine-induced astrogliosis through a blockade of OSMR/gp130 signaling and STAT3 phosphorylation. Exp Neurol 254:180-189, 2014

42. Ron D, Harding HP: Protein-folding homeostasis in the endoplasmic reticulum and nutritional regulation. Cold Spring Harb Perspect Biol 4:4, 2012

43. Rubovitch V, Shachar A, Werner H, Pick CG: Does IGF-1 administration after a mild traumatic brain injury in mice activate the adaptive arm of ER stress? Neurochem Int 58:443-446, 2011

44. Sadik G, Tanaka T, Kato K, Yamamori H, Nessa BN, Morihara T, et al: Phosphorylation of tau at Ser214 mediates its interaction with 14-3-3 protein: implications for the mechanism of tau aggregation. J Neurochem 108:33-43, 2009

45. Shoulders MD, Ryno LM, Genereux JC, Moresco JJ, Tu PG, Wu C, et al: Stress-independent activation of XBP1s and/or ATF6 reveals three functionally diverse ER proteostasis environments. Cell Reports 3:1279-1292, 2013

46. Small GW, Kepe V, Siddarth P, Ercoli LM, Merrill DA, Donoghue N, et al: PET scanning of brain tau in retired National Football League players: preliminary findings. Am J Geriatr Psychiatry 21:138-144, 2013

47. Smith DW, Bailes JE, Fisher JA, Robles J, Turner RC, Mills JD: Internal jugular vein compression mitigates traumatic axonal injury in a rat model by reducing the intracranial slosh effect. Neurosurgery 70:740-746, 2012

48. Song J, Park KA, Lee WT, Lee JE: Apoptosis signal regulating kinase 1 (ASK1): potential as a therapeutic target for Alzheimer's disease. Int J Mol Sci 15:2119-2129, 2014

49. Sosa MA, De Gasperi R, Paulino AJ, Pricop PE, Shaughness MC, Maudlin-Jeronimo E, et al: Blast overpressure induces shear-related injuries in the brain of rats exposed to a mild traumatic brain injury. Acta Neuropathol Commun 1:51, 2013

50. Stein TD, Alvarez VE, McKee AC: Chronic traumatic encephalopathy: a spectrum of neuropathological changes following repetitive brain trauma in athletes and military personnel. Alzheimers Res Ther 6:4, 2014

51. Stern RA, Riley DO, Daneshvar DH, Nowinski CJ, Cantu $\mathrm{RC}, \mathrm{McKee} \mathrm{AC}$ : Long-term consequences of repetitive brain trauma: chronic traumatic encephalopathy. PM R 3 (10 Suppl 2):S460-S467, 2011

52. Stutzbach LD, Xie SX, Naj AC, Albin R, Gilman S, Lee VM, et al: The unfolded protein response is activated in diseaseaffected brain regions in progressive supranuclear palsy and Alzheimer's disease. Acta Neuropathol Commun 1:31, 2013

53. Turner RC, Lucke-Wold BP, Robson MJ, Omalu BI, Petraglia AL, Bailes JE: Repetitive traumatic brain injury and development of chronic traumatic encephalopathy: a potential role for biomarkers in diagnosis, prognosis, and treatment? Front Neurol 3:186, 2012

54. Turner RC, Naser ZJ, Logsdon AF, DiPasquale KH, Jackson GJ, Robson MJ, et al: Modeling clinically relevant blast parameters based on scaling principles produces functional \& histological deficits in rats. Exp Neurol 248:520-529, 2013 
55. Watanabe T, Elovic E, Zafonte R: Chronic traumatic encephalopathy. PM R 2:671-675, 2010

56. Yang CC, Kuai XX, Li YL, Zhang L, Yu JC, Li L, et al: Cornel iridoid glycoside attenuates tau hyperphosphorylation by inhibition of PP2A demethylation. Evid Based Complement Alternat Med 2013:108486, 2013

57. Ye J, Rawson RB, Komuro R, Chen X, Davé UP, Prywes R, et al: ER stress induces cleavage of membrane-bound ATF6 by the same proteases that process SREBPs. Mol Cell 6:13551364,2000

58. Yokobori S, Mazzeo AT, Hosein K, Gajavelli S, Dietrich WD, Bullock MR: Preconditioning for traumatic brain injury. Transl Stroke Res 4:25-39, 2013

59. Zhou S, Yin X, Zheng Y, Miao X, Feng W, Cai J, et al: Metallothionein prevents intermittent hypoxia-induced cardiac endoplasmic reticulum stress and cell death likely via activation of Akt signaling pathway in mice. Toxicol Lett 227:113-123, 2014

60. Zhu X, Zhang J, Sun H, Jiang C, Dong Y, Shan Q, et al: Ubiquitination of inositol-requiring enzyme 1 (IRE1) by the E3 ligase CHIP mediates the IRE1/TRAF2/JNK pathway. J Biol Chem 289:30567-30577, 2014

\section{Disclosure}

Dr. Lee is a consultant for UpToDate and a patent holder with Cornelli Consulting.

\section{Author Contributions}

Conception and design: Lucke-Wold. Acquisition of data: LuckeWold, Logsdon, Nguyen. Analysis and interpretation of data: Lucke-Wold, Logsdon, Nguyen. Drafting the article: LuckeWold. Critically revising the article: all authors. Reviewed submitted version of manuscript: all authors. Approved the final version of the manuscript on behalf of all authors: Rosen. Statistical analysis: Lucke-Wold. Administrative/technical/material support: Rosen, Bailes, Lee, Omalu. Study supervision: Rosen.

\section{Correspondence}

Charles L. Rosen, Department of Neurosurgery, West Virginia University School of Medicine, One Medical Center Dr., P.O. Box 9183 Health Sciences Center, Morgantown, WV 265069183.email: crosen@hsc.wvu.edu. 